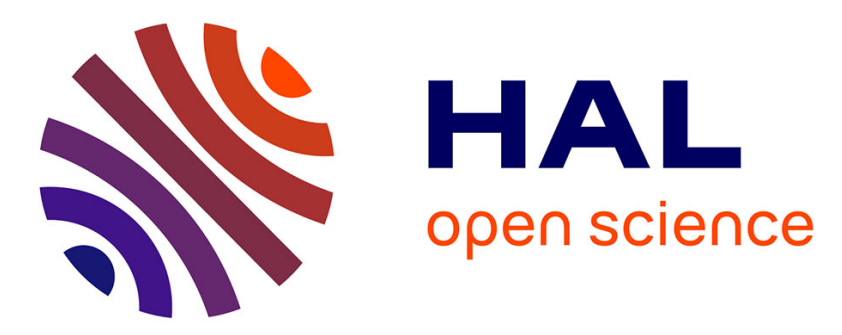

\title{
Description and experimental results of a panoramic K-Band radar dedicated to perception in mobile robotics applications
}

\author{
R. Rouveure, P. Faure, M.O. Monod
}

\section{- To cite this version:}

R. Rouveure, P. Faure, M.O. Monod. Description and experimental results of a panoramic K-Band radar dedicated to perception in mobile robotics applications. Journal of Field Robotics, 2017, pp.1-27. 10.1002/rob.21770 . hal-02607276

\section{HAL Id: hal-02607276 \\ https://hal.inrae.fr/hal-02607276}

Submitted on 16 May 2020

HAL is a multi-disciplinary open access archive for the deposit and dissemination of scientific research documents, whether they are published or not. The documents may come from teaching and research institutions in France or abroad, or from public or private research centers.
L'archive ouverte pluridisciplinaire $\mathbf{H A L}$, est destinée au dépôt et à la diffusion de documents scientifiques de niveau recherche, publiés ou non, émanant des établissements d'enseignement et de recherche français ou étrangers, des laboratoires publics ou privés. 


\title{
Description and Experimental Results of a Panoramic K-Band Radar Dedicated to Perception in Mobile Robotics Applications
}

Raphaël Rouveure, Patrice Faure and Marie-Odile Monod

National Research Institute of Science and Technology for Environment and Agriculture (Irstea), Technologies and Information Systems Research Unit (TSCF), Clermont-Ferrand Regional Center, 9 avenue Blaise Pascal, CS 20085, F-63178 Aubière, France

Email addresses: raphael.rouveure@irstea.fr; patrice.faure@irstea.fr; marie-odile.monod@irstea.fr Correspondence should be addressed to Raphäl Rouveure, raphael.rouveure@irstea.fr

\begin{abstract}
Robust environmental perception is a crucial parameter for the development of autonomous ground vehicle applications, especially in the field of agricultural robotics which is one of the priorities for the Horizon 2020 robotics funding (EU funding program for research and innovation). Because of uncontrolled and changing environmental conditions in outdoor and natural environments, data from optical sensors classically used in mobile robotics can be compromised and unusable. In such situations, microwave radar can provide an alternative and complementary solution for perception tasks. The aim of this paper is to present PELICAN radar, a microwave radar specifically designed for mobile robotics applications, including obstacle detection, mapping and situational awareness in general. PELICAN radar provides each second a view over $360^{\circ}$ of the surrounding environment, from $5 \mathrm{~m}$ up to $100 \mathrm{~m}$. A description of the technological solutions adopted for the development of PELICAN radar (hardware and software) is presented. Results obtained in various outdoor and open environments, including urban and natural areas, are also described. Sets of PELICAN radar data are made available online for researchers interested in processing this type of data.
\end{abstract}

\section{Keywords}

Microwave radar; FMCW radar; mobile robotics; 2D imaging; robust perception

\section{Introduction}

Agriculture is a major economic sector in the European Union. This domain is changing constantly due to economical, societal or environmental constraints. Agriculture has to maintain a high level of agricultural production despite the decline year-to-year of the number of farmers. Agriculture has to improve its competitiveness at a global level, in order to deal with international competition and to develop new markets. And agriculture has to improve agricultural practices in order to reduce the impacts on the environment, to better conserve biodiversity and ensure good nutrition for all.

To address these challenges, robotics is one of the avenues explored (Emmi, Gonzalez-de-Soto, Pajares, \& Gonzalez-de-Santos, 2014; Slaughter, \& Downey, 2008; CARRE, n.d.; Amazone, 2009). Robotics should enable the agricultural sector to improve its performances in several domains simultaneously: an increase of the human's response capability in space and time; an improvement of agricultural work quality and productivity while preserving the environment; the preservation of workers' health and security. But if several research programs or industrial developments have shown the scientific and technological feasibility of robots to realize agricultural tasks in outdoor and open environments, many obstacles remain to be overcome. 
A major problem for the development of Autonomous Ground Vehicles (AGV) applications in outdoor and uncontrolled environments such as agricultural fields is related to the availability of robust sensors. The sensors used to understand the surrounding environment of the robots have to deal with complex and time-varying situations due to weather conditions (fog, rain, and snow), variations of ambient light level, day/night cycles or the presence of obscurants such as smoke or dust. These Degraded Visual Environment (DVE) conditions can affect the functioning of the optical sensors (laser and vision) that are classically used in robotics applications. At the limit, these sensors can be completely ineffective and the robots become blind.

Microwave radar sensors can provide alternative and complementary solutions for perception in outdoor environments in order to overcome the limitations of optical systems. Owing to a centimer or a millimeter wavelength, microwave radars are weakly disturbed by environmental conditions and they can provide robust information even in degraded visual conditions. Microwave radars are typically found in domains which require long range measurements and high resistance to weather conditions: military domain, aeronautical and space sectors, or navy for example. The agricultural domain must also deal with complex environments (low structured and time-varying environments, weather conditions, etc.), but economic and technological constraints impose low cost and small sizes for the development of new equipment. In that sense, radar technology must be progressively adapted for AGV applications in the agricultural domain, in terms of dimension, weight, energy consumption, performances and cost (Brooker \& Jonhson, 2015).

Microwave radars are developed and used for several years for mobile robotics applications. Radar imagers are used for visualization and scene classification (Brooker, Jonhson, Underwood, Martinez, \& Xuan, 2015; Brooker et al., 2006; Reina, Milella, \& Underwood, 2012; Reina, Underwood, Brooker, \& Durrant-Whyte, 2011), for 2D/3D mapping of the environment or for Simultaneous Localization And Mapping (SLAM) applications (Foessel, Bares, \& Whittaker, 2001; Adams \& Jose, 2012; Jaud, Rouveure, Moiroux-Arvis, Faure, \& Monod, 2014). Researchers are also interested in the fusion of radar and vision data for obstacle detection and environment reconstruction, considering the complementarity between radar sensors (depth detection ability and robustness to environmental conditions) and vision sensors (high spatial resolution, color and texture analysis) (Wang, Zheng, Xin, \& Ma, 2011; El Natour, Ait-Aider, Rouveure, Berry, \& Faure, 2015; Reina, Milella, \& Rouveure, 2015; Milella, Reina, Underwood, \& Douillard, 2014). In the more specific domain of automotive applications, radars are used for Adaptive Cruise Control (ACC) (Zeng \& Deng, 2015; Tokoro, Kuroda, Kawakubo, Fujita, \& Fujinami, 2003), for the detection of moving and static targets (cars, pedestrians) (Hasch et al., 2012; Heuer, Al-Hamadi, Rain, \& Meinecke, 2014; Heuel \& Rohling, 2012) or for the estimation of road geometry (Hernandez-Gutierrez, Nieto, Bailey, \& Nebot, 2011). The development of research activities around radar sensors can be hampered by the limited number of radar systems available on the market. In the automotive domain, radar sensors such as the AC20 from TRW Autocruise Company or the Mid Range Radar (MRR) sensor from Bosch Company are widely distributed and they equip trucks or passenger vehicles; but their use outside the field for which they were originally designed is difficult considering their specific design configuration. The I200 radar from Navtech Company is a $77 \mathrm{GHz}$ radar scanner (Navtech, 2014). It is particularly used in Port Container Terminal as a part of motion control and navigation of straddle carriers. It has also been used as an obstacle detection sensor by the Red Team Vehicle during the 2005 DARPA Grand Challenge. In the academic field, several laboratories have designed their own radar sensor. The Carnegie Mellon University (CMU) in USA has designed a $77 \mathrm{GHz}$ radar for obstacle avoidance and road navigation in heavy-dust conditions (Foessel et al., 2001). This two-axis bistatic radar, equipped with a pencil beam antenna, is able to build 3D representation of the environment. The Nanyang Technological University (NTU) in Singapore has also developed a $77 \mathrm{GHz}$ radar. It is a two-axis 
mirror scanner, mainly devoted for simultaneous localization and mapping applications (Adams \& Jose, 2006). And the Australian Center for Field Robotics (ACFR) has developed several radar scanners in the past few years. Operating at $77 \mathrm{GHz}$ or $93 \mathrm{GHz}$, these radars were originally intended for mining applications (Widzyk-Capehart et al., 2006). The potential of these systems have led to their use in mobile robotics applications such as terrain visualization (Brooker et al., 2006), navigation aid (Brooker et al., 2015; Reina, Jonhson, \& Underwood, 2015) or scene interpretation (Reina et al., 2012; Milella, Reina, \& Underwood, 2015).

PELICAN radar was designed and developed by Irstea Institute. General views of PELICAN radar are presented in Figure 1.

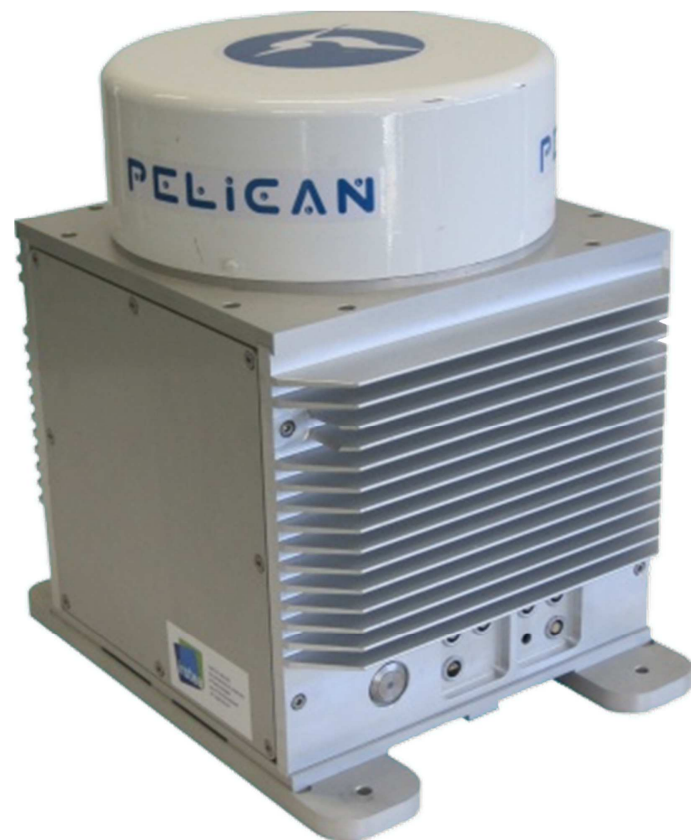

(a)

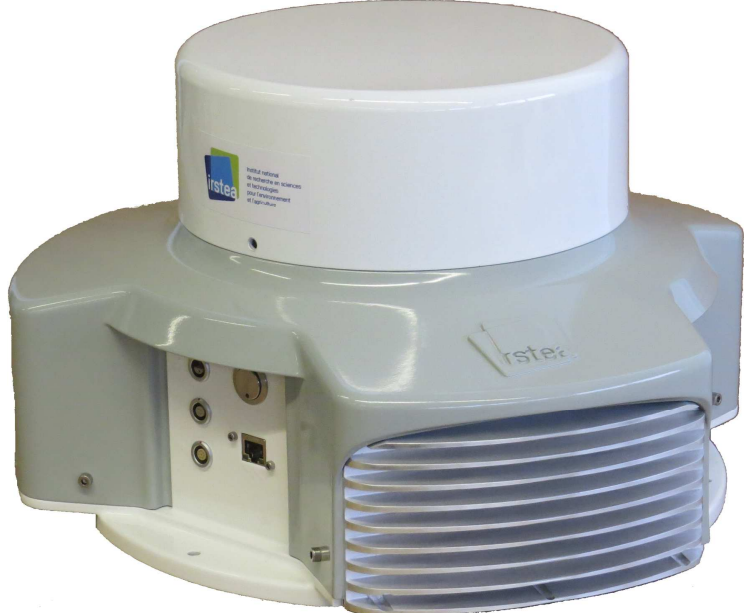

(b)

Figure 1: General view of PELICAN radar, considering two different housings. (a) Initial housing made of aluminum. (b) The second version of the housing has been realized with a $3 \mathrm{D}$ printer.

PELICAN radar is a microwave perception system specifically developed for mobile robotics applications. It is based on frequency-modulated continuous-wave (FMCW) technology, which appears to be well adapted to mobile robotics applications. With pulse radars, the transmission duration $\tau_{\mathrm{d}}$ defines a blind zone from range zero to range $r_{\mathrm{d}}=c \tau_{\mathrm{d}} / 2$ (with $c$ light velocity). For autonomous robots applications requiring radar-target distance measurements over short distances, a large value of $r_{\mathrm{d}}$ can lead to unacceptable configuration. Thus, a major problem with pulse radars is to be able to concentrate over short time a very high peak power. Frequency-modulated continuous-wave radar provides an alternative solution, because it eliminates the blind zone introduced by the pulse duration in the case of pulse radars, and because the bandwidth of the transmitted signal can provide satisfactory distance resolutions.

The major limitation of FMCW radars is related to their low range distance measurements, typically lower than $1 \mathrm{~km}$. Transmission and reception of FMCW radar signals are achieved simultaneously, and the coupling between transmitting and receiving stages limits the transmitted power and thus the maximum range. But it is not a constraint in our application considering the envisaged radar-target distances $(\ll 1 \mathrm{~km})$. Moreover, the relative simplicity of FMCW architectures can help to develop small-sized systems, compatible with lightweight robots. 
PELICAN radar is equipped with an antenna which rotates in the horizontal plane and provides panoramic views over $360^{\circ}$ of the surrounding environment. The antenna is a directional fan-beam antenna, characterized by elevation and azimuth angles $\left(\Theta_{\mathrm{el}}, \Theta_{\mathrm{az}}\right)$. The fan-beam antenna produces a main beam with a narrow beamwidth in the horizontal dimension (the azimuth, $\Theta_{\mathrm{az}}$ ) and a large beamwidth in the vertical dimension (the elevation, $\Theta_{\mathrm{el}}$ ). An illustration is presented in Figure 2. The elevation and azimuth angles $\left(\varphi_{\mathrm{el}}, \varphi_{\mathrm{az}}\right)$ of a target within the antenna beam are not measured: a target $T_{\mathrm{r}}$ located at distance $r$ from de radar will be detected as point $T_{\mathrm{d}}$ on the main axis of the antenna, with polar coordinates $(\theta, r)$ in the $\left(X_{\mathrm{r}}, y_{\mathrm{r}}, Z_{\mathrm{r}}\right)$ robot-fixed reference frame. Such antennas are widely used in military or aeronautics domains (aircraft surveillance), and in marine domain (ships localization, anticollision).

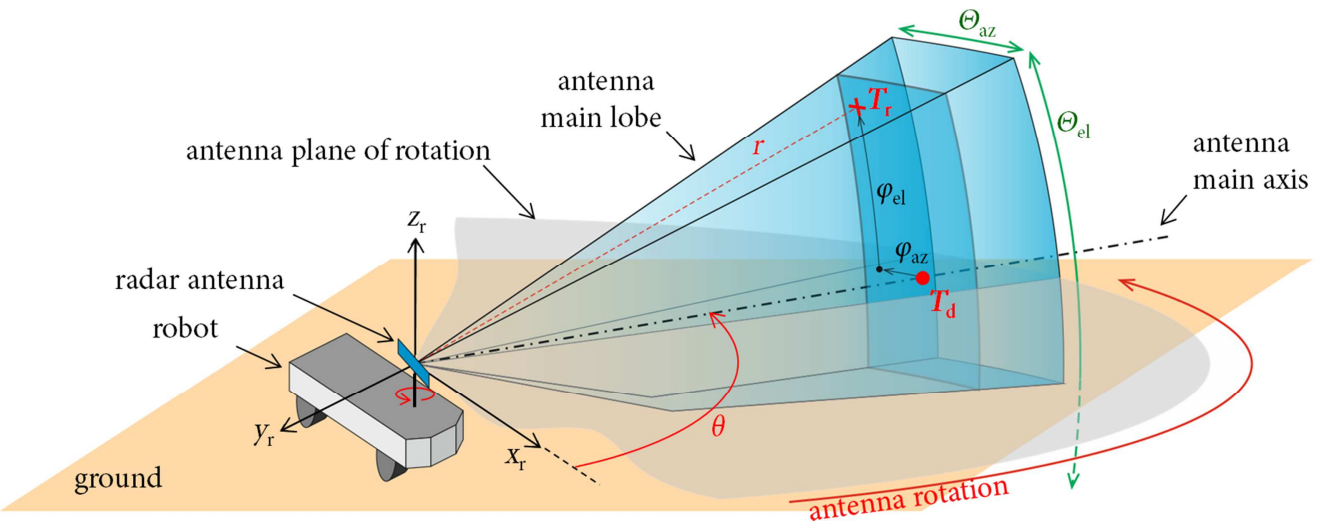

Figure 2: PELICAN radar measurement configuration. The main lobe of the fan-beam antenna is characterized by elevation and azimuth angles $\left(\Theta_{\mathrm{el}}, \Theta_{\mathrm{az}}\right)$. The antenna rotates in the horizontal (azimuth) plane.

The use of a fan-beam antenna is a main characteristic of PELICAN radar, and simultaneously a major limitation:

- On the one hand, the large elevation beamwidth of the antenna pattern makes the radar robust to the variations in pitch and roll of the robot. It is an interesting and desired feature when considering robot navigation in natural and non-flat environments.

- On the other hand, the elevation angle of a target within the antenna beam is unknown, so the altitude or the height of the target cannot be determined. The major consequence is that PELICAN radar can only build 2D images of the surrounding environment.

PELICAN radar is a Real Aperture Radar (RAR), because the angular resolution is determined by the real aperture of the antenna, in contrast to Synthetic Aperture Radar (SAR) typically used in airborne or spatial remote sensing systems. With RAR systems, the angular resolution can lead to a very coarse spatial resolution for long range applications. But this problem is not a limitation for the short range applications we are interested in. As the antenna rotates, radar signals are measured and radar spectra are computed at each degree of antenna rotation. And a complete panoramic radar image over $360^{\circ}$ is obtained at the end of each antenna revolution, with the concatenation of 360 radar spectra.

This paper is devoted for a detailed description of PELICAN radar and for experimental results. Section 2 is an overview of theoretical elements of PELICAN radar. Section 3 details the structure of PELICAN radar: microwave architecture, linear modulation of the transmitted signal, reception and processing of the backscattered radar signal. Section 4 is dedicated to the image construction process, and Section 5 is a specific focus on angular and distance resolutions improvement. Experimental results obtained in various environments (urban, semi-urban, natural area) are presented in Section 6. And Section 7 concludes the paper. 


\section{Theoretical Elements for PELICAN Radar}

PELICAN radar is based on FMCW principle. With FMCW radars, the transmitted signal is frequency modulated. The received signal is mixed with a part of the transmitted signal. The resulting signal is filtered and amplified in order to isolate the useful signal called beat signal $s_{b}$.

The transmitted signal of PELICAN radar is linearly modulated over a period $t_{\mathrm{m}}=1 / f_{\mathrm{m}}$ with a sawtooth function, with a sweep frequency $\Delta f$ centered about $f_{0}$. The principle of a sawtooth modulation is illustrated graphically in Figure 3.

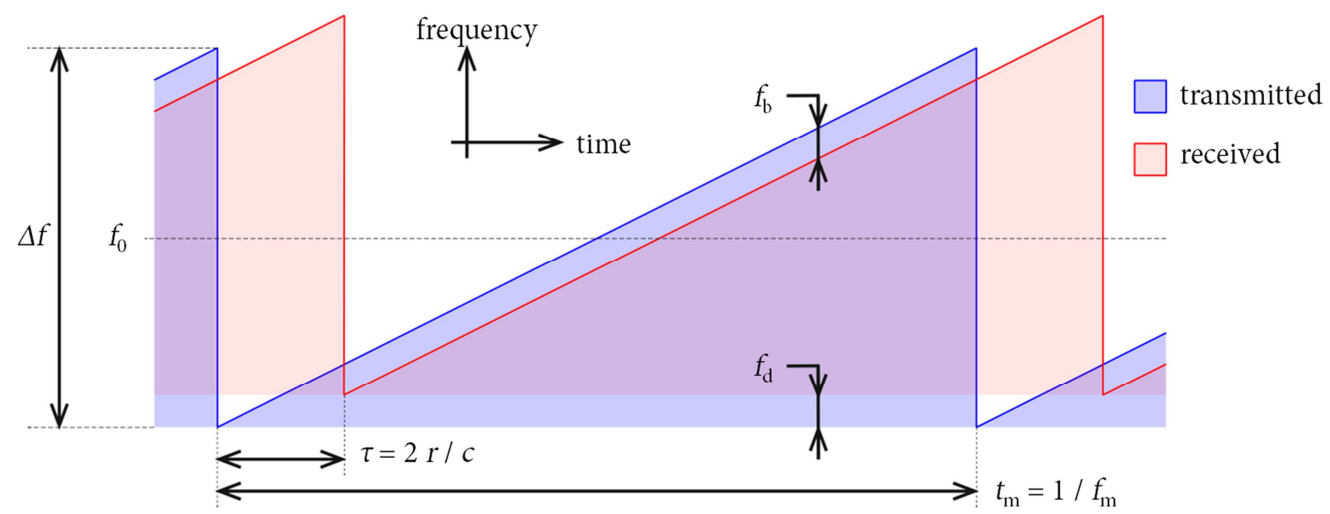

Figure 3: FMCW principle with a sawtooth modulation. Transmitted and received frequency vs. time functions appear in blue and red respectively. When a target is located at range $r$ with radial velocity $v_{\mathrm{r}}$, the received signal highlights a time delay $\tau$ corresponding to the radar-target distance $r$, and a vertical shift due to the frequency Doppler $f_{\mathrm{d}}$ introduced by $v_{\mathrm{r}}$.

Let us consider $i$ targets located at distance $r_{i}$ from the radar with radial velocities $V_{\mathrm{r} i}$. In that case, the beat signal $s_{b}$ which appears at the output of the radar can be written as (Rouveure, Faure, \& Monod, 2016; Skolnik, 1980; Monod, 1995)

$$
s_{\mathrm{b}}(t)=k_{\mathrm{m}} \sum_{i} a_{\mathrm{t}} a_{\mathrm{r} i} \cos (2 \pi \underbrace{\left(2 \Delta f f_{\mathrm{m}} \frac{r_{i}}{c}+2 f_{0} \frac{V_{\mathrm{r} i}}{c}\right)}_{f_{\mathrm{b} i}} t+\Phi_{i}),
$$

where $a_{\mathrm{t}}$ is the amplitude of transmitted signal, $a_{\mathrm{r} i}$ and $\Phi_{i}$ respectively the amplitude and a phase term of the signal received from target $i$, and $k_{\mathrm{m}}$ a mixer coefficient. The beat signal $s_{\mathrm{b}}$ is the sum of $i$ frequency components $f_{b i}$ (plus a phase term $\Phi_{i}$ ), each of them corresponding to a particular target $i$

$$
f_{\mathrm{b} i}=\underbrace{\left(2 \Delta f f_{\mathrm{m}} \frac{r_{i}}{c}\right)}_{f_{\mathrm{r}}}+\underbrace{\left(2 f_{0} \frac{V_{\mathrm{r} i}}{c}\right)}_{f_{\mathrm{d}}} .
$$

The first part $f_{\mathrm{r}}$ of Eq. (2) only depends on the range $r_{i}$, and the second part $f_{\mathrm{d}}$ is the Doppler shift induced by the radial velocity $V_{\mathrm{r} i}$. If $V_{\mathrm{r} i}=0$, one can see that $f_{\mathrm{b} i}$ is proportional to the radar-target distance $r_{i}$.

Expression (2) highlights a range-velocity ambiguity: with the measurement of the beat frequency, it is not possible to determine the radar-target distance without a priori knowledge of the radial velocity (and vice versa). This limitation can be avoided with a static environment assumption and the use of a proprioceptive sensor to measure the velocity of the vehicle. By combining the velocity $v$ of the vehicle with the antenna pointing direction $\theta$ (i.e. the angle between the direction of the vehicle and 
the antenna direction of propagation) measured with the antenna encoder, one can estimate the radial velocity $v_{\mathrm{r}}$ and the corresponding Doppler shift $f_{\mathrm{d}}$

$$
f_{\mathrm{d}}=\frac{2 f_{0}}{c} \underbrace{(v \cos (\theta))}_{V_{\mathrm{r}}} .
$$

From Eq. (2) and Eq. (3), the radar-target distance $r$ can be estimated

$$
r=\frac{c\left(f_{\mathrm{b}}-f_{\mathrm{d}}\right)}{2 \Delta f f_{\mathrm{m}}} .
$$

The corresponding range resolution $\delta r$ is given by the well-known formula

$$
\delta r=\frac{c}{2 \Delta f}
$$

which provides the relationship between the signal bandwidth $\Delta f$ and the range resolution $\delta r$. Equation (5) indicates that $\delta r$ only depends on $\Delta f$. an improvement of the range resolution is only obtained with an increase of the sweep frequency.

In Eq. (1), one can see that the amplitude of the beat signal $s_{b}$ is proportional to the term $\left(a_{t} a_{r i}\right)$ for target $i$. Assuming that the amplitude $a_{\mathrm{t}}$ of the transmitted signal is constant, the amplitude of $s_{b}$ is proportional to the amplitude $a_{\mathrm{r} i}$ of the received signal. We can use the simple form of the radar equation (Skolnik, 1980, Section 1.2, p. 3) to describe the parameters that affect $a_{\mathrm{r} i}$

$$
p_{\mathrm{r}}=\frac{p_{\mathrm{t}} G^{2} \lambda^{2} \sigma}{(4 \pi)^{3} r^{4}}
$$

with $p_{\mathrm{r}}$ and $p_{\mathrm{t}}$ received and transmitted power, $\lambda$ wavelength, $G$ antenna gain (monostatic case, i.e. the same antenna is used for transmission and reception) and $\sigma$ radar cross section of the target. The radar cross section (RCS) $\sigma$, expressed in meter square $\left(\mathrm{m}^{2}\right)$, is a measure of the degree of visibility of the target to the radar i.e. how a target re-radiates the energy of the incident radar signal. $\sigma$ depends on radar characteristics (wavelength, polarization) and on intrinsic parameters of the target: size, surface roughness, nature of constituting materials. It also depends on the orientation of the target to the radar. Considering that $p_{\mathrm{t}}, G$ and $\lambda$ are constant, $a_{\mathrm{r} i}$ is proportional to the square root of $\sigma$ and inversely proportional to the square of $r$

$$
a_{\mathrm{r} i} \propto \frac{\sqrt{\sigma}}{r^{2}}
$$

Generally, radar systems integrate range compensation electronics in order to cancel the term $r^{-2}$. Thus, the power of the received signal will be independent of range and will be only a function of $\sigma$.

\section{Description of PELICAN Radar}

A functional block diagram of PELICAN radar is presented in Figure 4. PELICAN radar includes a radar front-end (microwave components), a modulation module to control the frequency linearity of the transmitted signal, and a reception module to isolate the useful beat signal $s_{b}$. 


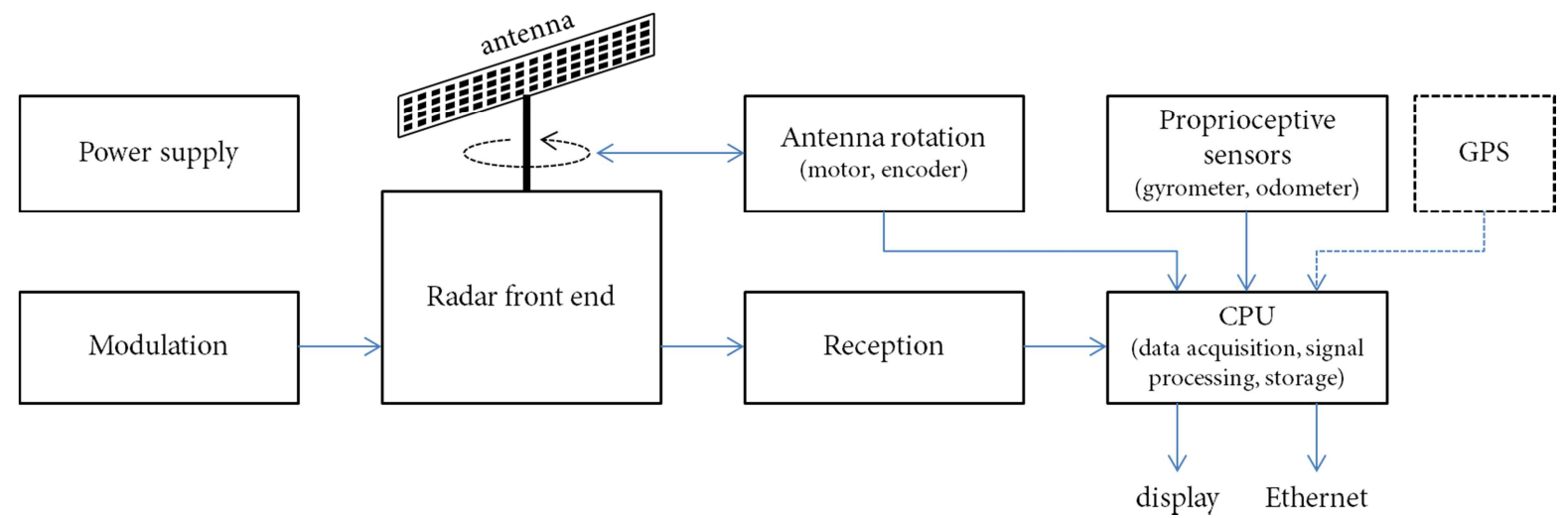

Figure 4: Functional block diagram of PELICAN radar.

The radar antenna is rotated by a direct current motor. The angular position of the antenna is measured with a 4096-point rotary digital encoder, allowing a precision of about $0.09^{\circ}$. Two proprioceptive sensors are used to take into account the motion (translation + rotation) of the vehicle on which the radar is positioned, and to correct the Doppler shift introduced by the vehicle velocity: a gyrometer and an odometer. The gyrometer is integrated inside the radar housing, and the odometer is a cable connected external sensor. A GPS can also be connected to PELICAN radar in order to know the position of the radar in an absolute terrestrial reference frame. Data acquisition and signal processing are based on a Pentium M-2 GHz PC/104 processor card. The PC/104 architecture has been selected due to its compact dimensions, and because it exhibits a good resistance to temperature variations. Raw or processed data can be stored internally on a flash disk, and/or transmitted for visualization and further treatment using an Ethernet link.

All components are integrated in the same housing. Figure 1 shows two versions of the housing, the first one made of aluminum and the second one realized in plastic with a $3 \mathrm{D}$ printer. The overall dimensions and weight of both versions of PELICAN radar are summarized in Table 1.

Table 1: Dimensions and weigh of PELICAN radar.

\begin{tabular}{lcc}
\hline & Aluminum version & 3D printer version \\
\hline Dimensions $(\mathrm{cm})$ & $\begin{array}{c}29-24-30 \\
\text { (length - width - height) }\end{array}$ & \begin{tabular}{c} 
(diameter - height) \\
\hline Weight $(\mathrm{kg})$
\end{tabular} \\
\hline
\end{tabular}

\subsection{Radar Front-End}

Figure 5 provides a schematic block diagram of PELICAN radar front-end. The transmitted signal is linearly modulated (mark $A$ ) and transmitted over the air by the antenna. PELICAN radar operates in monostatic configuration, i.e. the same antenna is used both for transmission and reception: a circulator isolates the transmission signal and the reception signals. As the antenna rotates, it is necessary to use a rotary joint in order to transmit the microwave signal to the antenna and to receive the backscattered signals from the antenna. Received signals are then mixed to a portion of the transmitted signal taken from a coupler. 


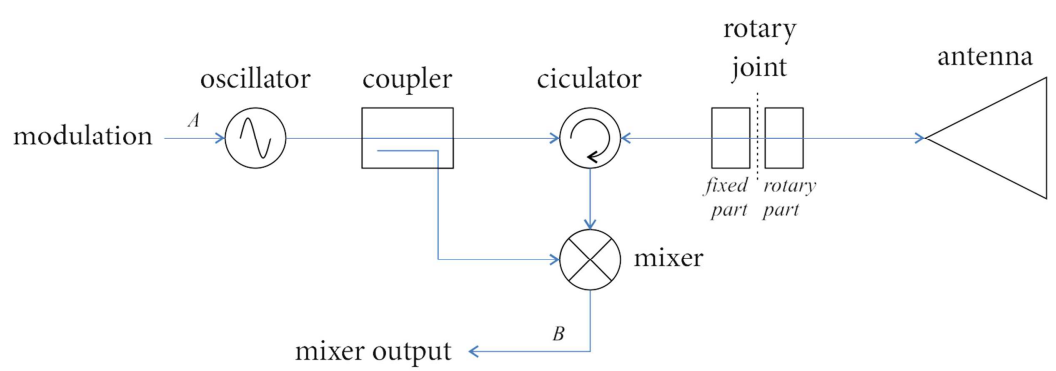

Figure 5: Schematic block diagram of PELICAN radar front-end.

The output of the mixer (mark $B$ ) is a complex signal, including dc component, transmitted signal, received signals, beat and sum signals, intermodulation sum and difference between received signals, and harmonics of higher order. A complete description of these signals can be found in Monod (1995) and Rouveure (2001). The output of the mixer is then amplified and filtered by the reception module presented in Section 3.3, in order to isolate the useful beat signal $s_{b}$.

As it can be seen in Eq. (1), the transmitted signal is characterized with four parameters:

- carrier frequency $f_{0}$,

- sweep frequency $\Delta f$,

- chirp repetition frequency $f_{\mathrm{m}}$,

- transmitted power $p_{\mathrm{t}}$ (or amplitude $a_{\mathrm{t}}$ ).

\subsubsection{Carrier Frequency $f_{0}$}

The choice of the carrier frequency $f_{0}$ of the transmitted signal is of major importance when defining and developing microwave radar systems. Numerous aspects must be taken into consideration in determining this carrier frequency (Rouveure et al., 2016). Within the framework of PELICAN radar, three predominant criteria have guided our choice:

- antenna parameters, which have a direct influence on radar dimensions and angular resolution. Indeed, the length $d$ of the antenna is inversely proportional to $f_{0}$; and the antenna beamwidth $\theta$ is inversely proportional to $f_{0}$ and $d$. A compromise has to be found between the size and the beamwidth of the antenna and the carrier frequency. The objective is to develop a compact radar sensor with a correct angular resolution, thus the weight and sizes of the radar must be carefully considered in order to be able to use it with various robots.

- the cost and the availability of microwave components (oscillator, antenna, etc.), which are still constraints for the development of new devices and applications in the industrial or research domains. It is a fluctuating situation, because due to the emergence of new markets, up-to-date components are regularly proposed by dealers.

- regulatory constraints. Devices and systems require authorizations to radiate RF energy. These authorizations are managed by national and international organizations (Agence Nationale des Fréquences, 2013; International Telecommunication Union, 2012). It is necessary to deal with limitations in the use of the radio-frequency spectrum, such as prohibited frequency bands, or bandwidth and power limitations.

The final choice has been a trade-off between these criteria, and we have decided to develop a K-band radar with a carrier frequency $f_{0}=24.125 \mathrm{GHz}$. This frequency belongs to the $24-24.25 \mathrm{GHz}$ industrial, scientific and medical (ISM) radio band, which is internationally reserved for industrial, scientific and medical purposes other than telecommunications. It must be noticed that devices which are using this band must tolerate interferences, because the band is free of use and it allows unlicensed operations. 


\subsubsection{Sweep Frequency $\Delta f$}

With FMCW radars, the frequency modulation of the transmitted signal provides a time reference of the received echoes, and allows distance determination. In Eq. (5), it can be seen that the range resolution $\delta r$ is only determined by the sweep frequency $\Delta f$. the higher the sweep frequency, the better the distance resolution. But the value of $\Delta f$ cannot be freely increased due to technological and regulatory constraints. On the one hand, the sweep frequency capabilities of microwave components are not infinite: for example, the maximum sweep frequency of an oscillator is typically defined as a small percentage of the carrier frequency; similarly, the bandwidth of the antenna is limited. On the other hand, the choice of the $24 \mathrm{GHz}$ ISM radio band to develop PELICAN radar imposes a bandwidth limitation: the ISM band is defined between $24 \mathrm{GHz}$ and $24.25 \mathrm{GHz}$, consequently the maximum allowed sweep frequency is $250 \mathrm{MHz}$. From Eq. (5), it comes that the optimal range resolution $\delta r$ is $0.6 \mathrm{~m}$. However, the maximum sweep frequency of the YIG (Yttrium Iron Garnet) oscillator used for PELICAN radar is limited to $195 \mathrm{MHz}$, and the corresponding range resolution $\delta r$ is $0.77 \mathrm{~m}$. Unfortunately, the transition of the transmitted frequency at the end of each modulation slope (from $f_{0}+\Delta f / 2$ to $\left.f_{0}-\Delta f / 2\right)$ introduces disturbances, and the effective range resolution $\delta r$ is given by

$$
\delta r=\frac{c}{2 k \Delta f}
$$

with $0 \leq k \leq 1$. The coefficient $k$ indicates that only $(k \times 100)$ per cent of the modulation period $t_{\mathrm{m}}$ is used to estimate the beat frequency. Considering PELICAN radar configuration, i.e. $\Delta f=195 \mathrm{MHz}$ and $k=0.73$, the effective range resolution is $\delta r=1.05 \mathrm{~m}$.

\subsubsection{Chirp Repetition Frequency $f_{\mathrm{m}}$}

The product between the sweep frequency $\Delta f$ and the chirp repetition frequency $f_{\mathrm{m}}$ defines the slope of the modulation law. The minimum value of $f_{\mathrm{m}}$ is determined by the dwell time, i.e. the time that the antenna beam spends on a point target. Considering an antenna velocity rotation $\omega$ (expressed in radian per second) and an antenna beamwidth $\theta$ (expressed in radian), the dwell time $t_{\mathrm{d}}$ is defined as

$$
t_{\mathrm{d}}=\frac{\theta}{\omega} .
$$

The point target must be observed for at least one period of the modulation, implying the following minimum value of $f_{\mathrm{m}}$

$$
f_{\mathrm{m}}=\frac{1}{t_{\mathrm{d}}}=\frac{\omega}{\theta}
$$

The antenna beamwidth $\theta$ of PELICAN radar is $5^{\circ}$ in the horizontal plane (see Section 3.1.5), and it has been decided to build one panoramic radar image per second (i.e. $\omega=60 \mathrm{rpm}=2 \pi \mathrm{rad} / \mathrm{s}$ ), thus we obtain a minimum chirp repetition frequency of $72 \mathrm{~Hz}$. With the aim of having one radar signal acquisition at each degree of antenna rotation, the chirp repetition frequency has been finally set at a value of $360 \mathrm{~Hz}$. One can notice that this value cannot be freely increased due to the maximum tuning speed of the oscillator, and to the bandwidth of the data acquisition device.

\subsubsection{Amplitude $a_{\mathrm{t}}$ of the Transmitted Signal}

From Eq. (6), it can be seen that the maximum detection distance is above all limited by the transmitted power $p_{t}$. For the mobile robotics applications being considered, the maximum distance 
measurements will be typically limited between one hundred and two hundred meters. Such distances allow the use of a low transmitted power $p_{\mathrm{t}}$, and they avoid the use of expensive microwave amplifiers.

Screenshots in Figure 6 show a spectrum analysis of the PELICAN radar transmitted signal done with an Agilent E4408B Spectrum Analyzer. The signal measurement is done without the antenna, with a cable connected to the output of the rotary joint (see Figure 5). It can be seen that the center frequency is $24.125 \mathrm{GHz}$, with a start frequency of $24.027 \mathrm{GHz}$ and a stop frequency of $24.222 \mathrm{GHz}$ (i.e. total sweep frequency $\Delta \mathrm{f}=195 \mathrm{MHz}$ ). The mean transmitted power is about $14.7 \mathrm{dBm}(29.5 \mathrm{~mW})$. One can see in Figure 6(b) that the transmitted power is not constant over the transmitted frequency: it highlights a sinusoidal-like variation, with a peak-to-peak variation of about $0.5 \mathrm{dBm}$. The spectral analysis provides the bandwidth of the transmitted signal, but it does not allow evaluating the linearity of the frequency modulation.

(a)

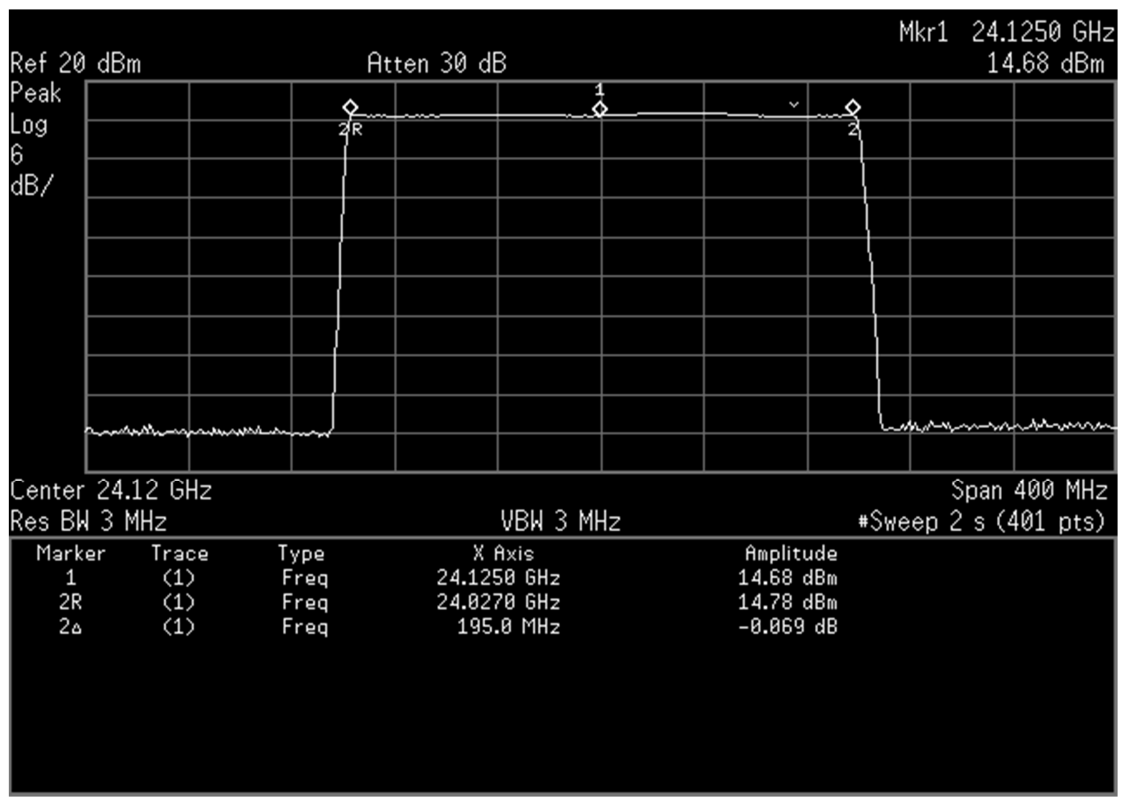

(b)

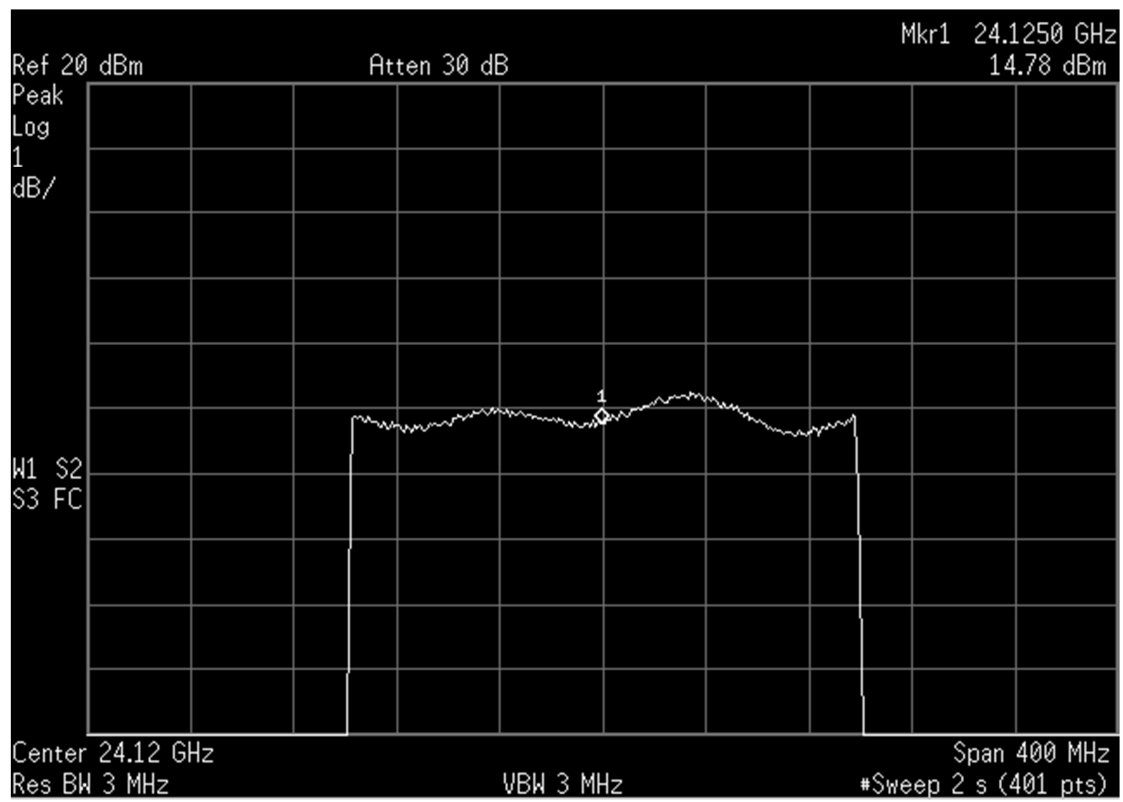

Figure 6: Spectral analysis of the transmitted signal. The measurements were carried out with an Agilent E4408B Spectrum Analyzer. (a) The transmitted signal is centered on $24.125 \mathrm{GHz}$, with a bandwidth of $195 \mathrm{MHz}$. (b) A zoom on the top of the curve shows that the power is not constant over the transmitted frequency and highlights a peak-to-peak variation of about $0.5 \mathrm{dBm}$. 
The parameters of the transmitted signal used with PELICAN radar are resumed in Table 2.

Table 2: PELICAN radar parameters.

\begin{tabular}{ll}
\hline $\begin{array}{l}\text { Carrier frequency } f_{0} \\
\text { (lengthwave } \lambda \text { ) }\end{array}$ & $\begin{array}{l}24.125 \mathrm{GHz} \\
(12.4 \mathrm{~mm})\end{array}$ \\
\hline $\begin{array}{l}\text { Sweep frequency } \Delta f \\
\text { (start-stop frequencies) }\end{array}$ & $195 \mathrm{MHz}$ \\
\hline Modulation frequency $f_{\mathrm{m}}$ & $(24.027-24.222 \mathrm{GHz})$ \\
\hline Transmitted power $p_{t}$ & $360 \mathrm{~Hz}$ \\
\hline
\end{tabular}

\subsubsection{Antenna Parameters}

The antenna specifications of PELICAN radar have been defined by Irstea Institute, and the design, simulation and manufacture of the antenna have been realized by ANTENNESSA manufacturer. PELICAN antenna is a planar array antenna (see Figure 7). It is composed of a rectangular array of $4 \times 18$ radiating elements. The antenna is fixed on an aluminum bracket with dielectric screws. The overall dimension of the antenna + bracket is $18.5 \times 5 \mathrm{~cm}$.

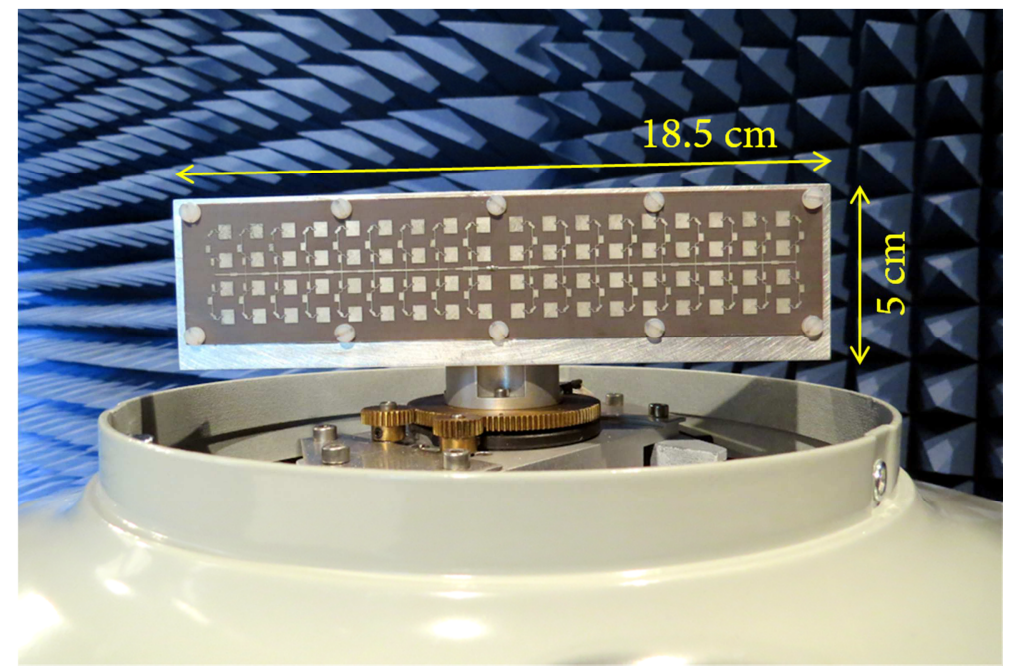

Figure 7: PELICAN planar array antenna. The antenna is composed of $4 \times 18$ radiating elements in a rectangular configuration. The antenna is fixed on an aluminum bracket with dielectric screws.

PELICAN antenna is a fan-beam antenna, which produces a main beam with a narrow beamwith in the horizontal plane (the azimuth) and a wider beamwidth in the vertical plane (the elevation). The antenna radiation pattern provided by the manufacturer is presented in Figure 8: the half-power beamwidth is $5^{\circ}$ in the azimuth dimension $\left(\Theta_{\mathrm{az}}\right)$, and $24.5^{\circ}$ in the elevation dimension $\left(\Theta_{\mathrm{el}}\right)$.

The antenna rotates in the horizontal plane over the full $360^{\circ}$ in order to build a panoramic image of the environment. The antenna is equipped with an encoder which measures the pointing direction $\theta$ in a radar-fixed reference frame. The detected elements are localized through (azimuth $\theta$, distance $r$ ) polar coordinates in the radar-fixed reference frame. Considering the chirp repetition frequency $f_{m}=360 \mathrm{~Hz}$ and the antenna velocity $\omega=60 \mathrm{rpm}$ (see section 3.1.3), 360 radar spectra are used to build one panoramic radar image. The antenna performances (beamwidth, gain) are resumed in Table 3 , considering the carrier frequency of $24.125 \mathrm{GHz}$. 
(a)

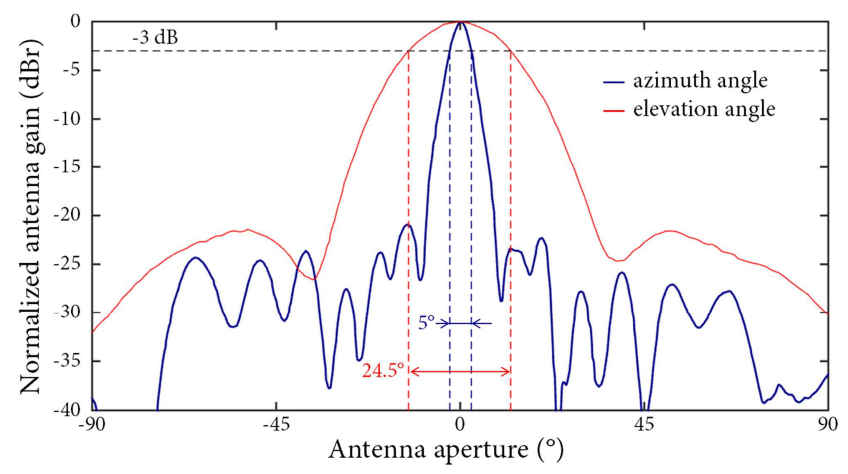

(b)

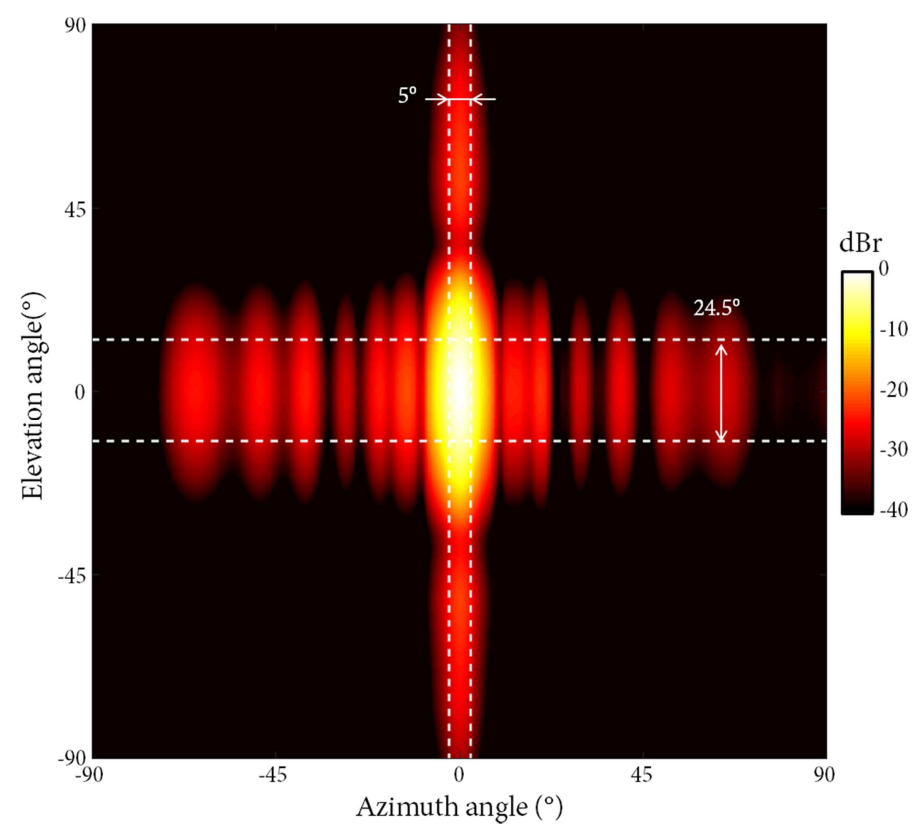

Figure 8: Radiation pattern of the PELICAN fan-beam antenna provided by ANTENNESA manufacturer. Half-power beamwidth: $5^{\circ}$ in the azimuth plane, $24.5^{\circ}$ in the elevation plane. (a) Normalized gain of the antenna along the main azimuth and elevation axis. (b) $3 \mathrm{D}$ antenna radiation pattern.

One can notice that the use of a fan-beam antenna is a main characteristic and a major limitation of PELICAN radar. The azimuth beamwidth defines the angular resolution. The use of a large elevation beamwidth is a purpose in order to make the radar robust to positioning variations in pitch and roll of the robot: owing to the size of the beam, a target will be detected even with variations in robot's attitude. But the use of a fan-beam antenna also implies several constraints. Firstly, the roll angle can introduce distortions during the panoramic images construction; but this distortion is not corrected because the elevation and azimuth angles $\left(\varphi_{\mathrm{el}}, \varphi_{\mathrm{az}}\right)$ of the targets are unknown (see Figure 2). Secondly, as the elevation angles $\varphi_{\mathrm{el}}$ are not measured, the elevations or heights of the targets cannot be determined, and the radar can only build $2 \mathrm{D}$ images of the environment. The $2 \mathrm{D}$ image construction is the projection of targets' echoes on the rotation plane of the antenna, which introduces a systematic overestimation of the ground distance excepted when $\varphi_{\mathrm{el}}=0^{\circ}$. This last distortion can be corrected with knowledge about the environment as described in Jaud, Rouveure, Faure, Monod, \& MoirouxArvis (2014). 
Table 3: PELICAN antenna characteristics.

\begin{tabular}{lll}
\hline Elevation aperture $\Theta_{\mathrm{el}}$ & $\begin{array}{l}-3 \mathrm{~dB} \text { beamwidth } \\
\text { Side lobe level }\end{array}$ & $\begin{array}{l}24.5^{\circ} \\
<-21.5 \mathrm{~dB}\end{array}$ \\
\hline Azimuth aperture $\Theta_{\mathrm{az}}$ & $-3 \mathrm{~dB}$ beamwidth & $5^{\circ}$ \\
& Side lobe level & $<-21.3 \mathrm{~dB}$ \\
\hline Gain $G$ & & $21.08 \mathrm{dBi}$ \\
\hline Rotation velocity $\omega$ & & $60 \mathrm{rpm}$ \\
& & $(2 \pi \mathrm{rad} / \mathrm{s})$ \\
\hline
\end{tabular}

\subsubsection{Minimum and Maximum Distances Measurement}

By comparison with "classical" radar applications such as radar remote sensing, mobile robotics applications represent complex configurations for radars. In radar remote sensing, the distances variations introduced by the position of the targets within the antenna footprint are low compared to the radar-targets distances, and the corresponding amplitude variations remain reduced. In mobile robotics applications, distance measurements are achieve from distances close to zero up to the maximum range: owing to the term $\mathrm{r}^{-2}$ in Eq. (7), the amplitude variations of the received signals are large, and the receiver electronics can be difficult to adjust. With FMCW radars, several factors are related to the minimum and maximum measurable distances.

\section{Minimum distance measurement}

FMCW principle theoretically allows distance measurements from null distance. But several factors limit this minimum distance.

The first factor is related to the minimum measurable beat frequency. If radar spectra are computed with the Fourier transform, the minimum beat frequency $f_{\text {min }}$ is determined by the modulation frequency $f_{\mathrm{m}}$, because at least one period of the signal must be observed

$$
f_{\mathrm{bmin}}=\frac{f_{\mathrm{m}}}{k}
$$

with $0 \leq k \leq 1$. The coefficient $k$ indicates that the spectral analysis can be done on a portion of the radar signal. Substituting Eq. (11) in Eq. (4) and considering a null radial velocity (i.e. $f_{\mathrm{d}}=0$ ), the minimum distance $r_{\min }$ is given by

$$
r_{\min }=\frac{c}{2 k \Delta f}
$$

One can observe that $r_{\min }$ is equivalent to the range resolution $\delta r$ presented in Eq. (8). Several values of $r_{\min }$ as a function of $\Delta f$ are presented in Table 4 . The minimum distance $r_{\min }$ is equal to $1.05 \mathrm{~m}$ with PELICAN radar configuration.

Table 4: Examples of minimum distance $r_{\min }$ for several values of sweep frequency $\Delta f$. PELICAN radar configuration is $\Delta f=195 \mathrm{MHz}$ and $k=0.73$.

\begin{tabular}{ccc}
\hline$\Delta f$ & \multicolumn{2}{c}{$r_{\min }(\mathrm{m})$} \\
$(\mathrm{MHz})$ & $k=1$ & $k=0.73$ \\
\hline 250 & 0.60 & 0.82 \\
\hline 195 & 0.77 & 1.05 \\
\hline 150 & 1.00 & 1.37 \\
\hline
\end{tabular}

A second factor that influences the minimum distance $r_{\min }$ is related to the shape of the transmitted signal. Figure 6(b) shows that the power of the oscillator is not constant over the transmitted 
frequency: the oscillator highlights a sinusoidal-like variation of the transmitted power as a function of the transmitted frequency, with a peak-to-peak variation of about $0.5 \mathrm{dBm}$. The shape of this amplitude modulation is unexpected: it is an intrinsic characteristic of the oscillator, and it will differ from one oscillator to another. By comparison with the received signals, the amplitude of this modulation can be high: the fundamental frequencies and their harmonics can prevent the detection of close targets. The spectral analysis of the amplitude modulation presented in Figure 6(b) indicates an occupied bandwidth up to $1.5 \mathrm{kHz}$, which corresponds to a theoretical obstacle located at range $2.8 \mathrm{~m}$. Low level reflected signals from targets situated below $2.8 \mathrm{~m}$ will be masked by this amplitude modulation of the transmitted signal.

The use of a radiating antenna introduces another limiting factor for the minimum measurable distance. Indeed, theoretical elements presented in Section 2 assume a free-space propagation model, where the received power decays as negative fourth power of the distance as it can be seen in Eq. (6). Free-space propagation model is only valid in the far field region of the antenna, also termed Fraunhofer region (Skolnik, 1980). For antenna physically larger than a half wavelength of the transmitted signal, the far field region is defined in terms of the Fraunhofer distance $r_{\mathrm{f}}$

$$
r_{\mathrm{f}}=\frac{2 d^{2}}{\lambda}
$$

where $d$ is the largest dimension of the antenna. Targets located at range $r \geq r_{\mathrm{f}}$ are considered belonging to the far field region. With the characteristics of PELICAN antenna, i.e. $\lambda=0.0125 \mathrm{~m}$ and $d=0.156 \mathrm{~m}$, we obtain a Fraunhofer distance $r_{\mathrm{f}} \approx 3.9 \mathrm{~m}$.

Considering all these limiting factors, the minimal measurement distance of PELICAN radar has been set at $5 \mathrm{~m}$.

\section{Maximum distance measurement}

Radar detection is based on signal detection. It means that a target will be detected if the power received from that target is superior to the mean value of the power noise. We introduced in that case the idea of minimum measurable power $p_{\text {rmin. }}$. With $p_{\text {rmin }}$ and Eq. (6), the maximum radar range $r_{\max }$ can be computed:

$$
r_{\max }=\left(\frac{p_{t} G^{2} \lambda^{2} \sigma}{(4 \pi)^{3} p_{\mathrm{rmin}}}\right)^{\frac{1}{4}}
$$

From Eq. (14), it can be seen that the increase of $r_{\max }$ is obtained with a reduction of the minimum measurable power $p_{\text {rmin }}$, or with an increase of the transmitted power $p_{t}$. This last solution is subject to technological limits: owing to the coupling between transmitting and receiving stages, the transmitted power is limited with FMCW radars. It is also subject to regulatory constraints such as power limitations.

As pulse radars, FMCW radars are subject to distance ambiguity. With pulse radars, the maximum distance before ambiguity is determined by the pulse repetition frequency (PRF): the echo from a target must be received before the transmission of the next pulse. The same phenomenon occurs with FMCW radars, and the maximum distance $r_{\max }$ before ambiguity is fixed by the modulation frequency $f_{\mathrm{m}}$

$$
r_{\max }=\frac{c}{2 f_{\mathrm{m}}} .
$$


When considering the modulation frequency of PELICAN radar $(360 \mathrm{~Hz}$, see Table 2), the maximum distance before ambiguity is $r_{\max }=417 \mathrm{~km}$, so we are sure to never reach this limit.

Another factor which limits the maximum range is the bandwidth of the data acquisition device. From Eq. (2), it can be seen that the higher the distance $r$, the higher the beat frequency $f_{\mathrm{b}}$. Substituting $f_{b}$ by $f_{b \max }$ in Eq. (4), and considering a null radial velocity (i.e. $f_{d}=0$ ), the maximum distance $r_{\max }$ is given by

$$
r_{\max }=\frac{c f_{\mathrm{bmax}}}{2 \Delta f f_{\mathrm{m}}} .
$$

The Nyquist-Shannon sampling theorem gives the theoretical relation between the maximum measurable frequency $f_{b \max }$ and the sampling rate $f_{s}$

$$
f_{\mathrm{bmax}} \leq \frac{f_{\mathrm{s}}}{2} .
$$

In order to apply low-pass anti-aliasing filter, we set the value of $f_{b \max }$ at $f_{\mathrm{s}} / 4$, and the corresponding maximum measurable distance is

$$
r_{\max }=\frac{c f_{\mathrm{s}}}{8 \Delta f f_{\mathrm{m}}},
$$

which corresponds to a maximum measurable distance of about $107 \mathrm{~m}$ with $f_{\mathrm{s}}=200 \mathrm{kHz}$ and PELICAN radar parameters described in Table 2.

\subsection{Modulation}

The formulas of the beat signal (Eq. (1)) and of the beat frequency (Eq. (2)) are theoretical relationships; they assume a perfect linear modulation of the transmitted signal. The objective of the modulator is to ensure that the transmitted frequency follows a linear variation law as described in Figure 3. The quality of this linear variation has a direct effect on the precision with which the beat frequency $f_{b}$, and hence range $r$, can be measured with FMCW radars. Here, two major factors are predominant:

- the spectral purity of the transmitted signal and

- the nonlinearity of the modulation law.

To transmit a radar signal as pure as possible, the choice of quality electronic components must be promoted. And to limit the nonlinearity of the modulation law, the radar developers should take care to the form of the modulation law during the design and development phases. The effect of a nonlinear transmitted signal is illustrated in Figure 9. When considering a theoretical linear modulation, the resulting beat frequency is constant for the whole modulation period (see Figure 9(top), dotted lines). If the modulation law is nonlinear (solid lines in Figure 9(top)), the resulting beat frequency will not be constant for the whole modulation period, with the introduction of spurious frequencies (Figure 9(bottom)). 


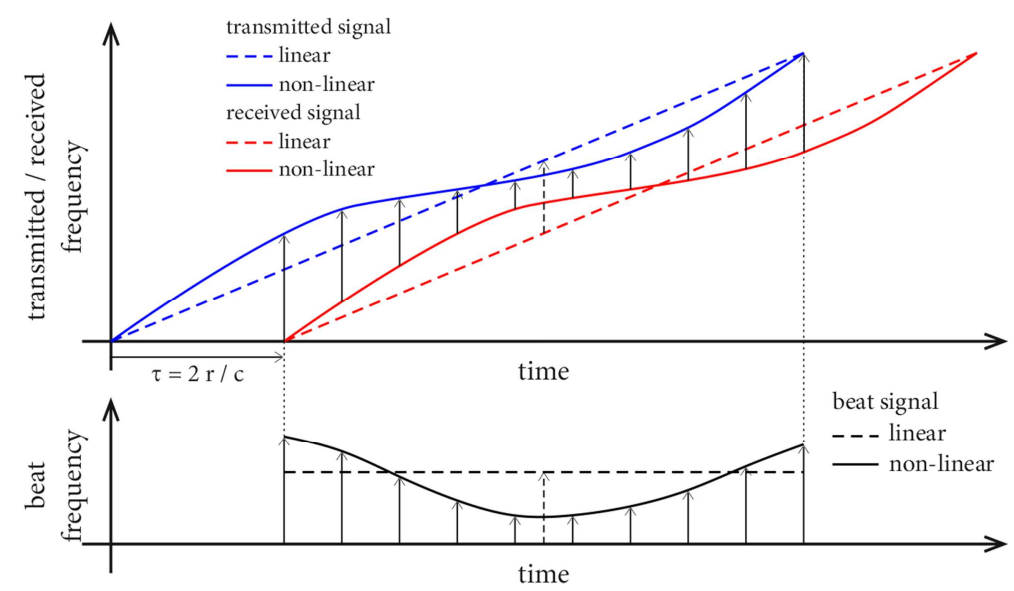

Figure 9: Effect of non-linearized transmitted signal considering a single target. Top: frequency vs. time of transmitted and received signals. Dotted lines: linear frequency modulation; solid lines: nonlinear frequency modulation. Bottom: frequency

vs. time of beat signal. With a linear frequency modulation, the beat frequency highlights a constant value during the modulation period (dotted line). The nonlinear modulation leads to a non-constant value of the beat frequency during the modulation period (solid line), and the introduction of spurious frequencies.

Methods for linearizing FMCW radar signal can be classified into two categories: open-loop and closed-loop methods. Closed-loop methods act continually during the radar signal transmission in order to ensure a continuous frequency lock. They offer higher linearity performances, but they also induce higher costs. They are particularly well-adapted to applications which require a wide bandwidth (Johnson \& Brooker, 2008; Alvestegui, 2011). Considering the necessary sweep frequency for our robotics applications and the use of a stable oscillator, we have selected an open-loop approach. In an open-loop arrangement, the modulation law is fixed, assuming a stable behavior of the oscillator over the time. Figure 10(top-left) is the relationship between the output frequency and the tuning signal of a given microwave oscillator. Also known as the tuning characteristic, this curve is a characteristic of the oscillator and varies from one oscillator to another. Figure 10(top-right) is the theoretical linear frequency variation which is expected. If a linear tuning signal is applied, we obtain a non-linear variation of the output frequency due to the shape of the tuning characteristic. Thus, it is necessary to apply the non-linear tuning signal shown in Figure 10(bottom) to take into account the tuning characteristic of the oscillator, and to obtain a linear frequency modulation of the radar signal. The shape of this tuning signal can be geometrically obtained: Figure 10(top-right) gives the expected frequency $f_{\mathrm{i}}$ at time $t_{\mathrm{i}}$; and Figure 10(top-left) provides the value $s_{\mathrm{i}}$ of the tuning signal to apply to obtain the frequency $f_{\mathrm{i}}$. From $t_{\mathrm{i}}$ and $s_{\mathrm{i}}$, the modulation law in Figure 10(bottom) can then be gradually constructed. 


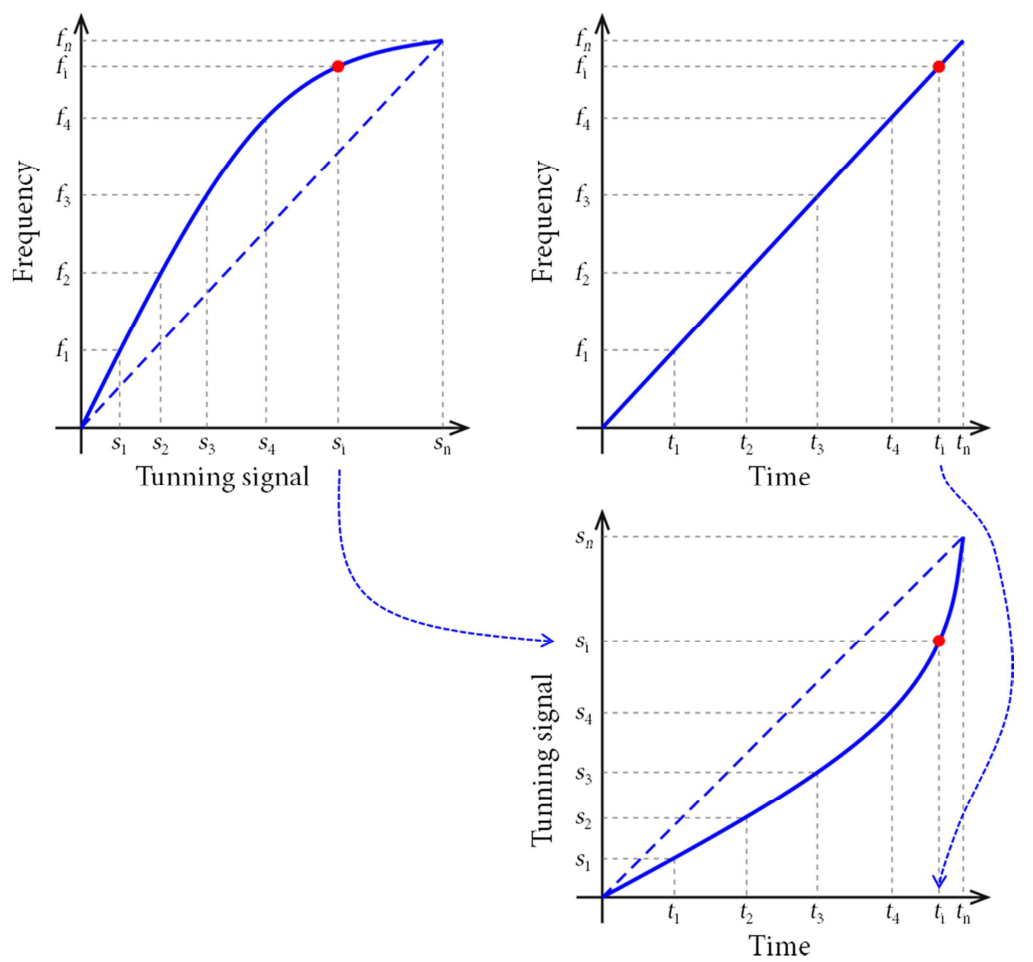

Figure 10: Principle of the open-loop linearization method. Top-left: the tuning characteristic is the relationship between the output frequency and the tuning signal. Top-right: expected frequency vs. time linear function. Bottom: the non-linear tuning signal vs. time function is used in order to obtain a linear frequency modulation of the radar signal. At time $t$, the expected frequency is $f_{\mathrm{i}}$ : the tuning signal $s_{\mathrm{i}}$ is applied at time $t_{\mathrm{i}}$ in order to transmit the frequency $f_{\mathrm{i}}$.

The tuning characteristic is obtained with the use of laboratory equipment (Agilent E4408B spectrum analyzer in our case). But this manual step by step procedure is not sufficient to obtain a correct linear frequency modulation of the transmitted signal, because the dynamic behaviors of the oscillator and of its driver are not taken into account. For that reason we have developed an approach which is based on the measurement of the nonlinearities of the beat frequency over the modulation period. In this approach (see Figure 11), a radar measurement is realized using a canonical target (trihedral corner or Luneburg lens). A time-frequency analysis of the measured beat signal is achieved in order to evaluate the variations of the beat frequency over the modulation period. The deviations $e_{i}$ of the beat frequency are used to gradually modify the modulation law:

- a small value $\delta s_{i}$ of the tuning signal, proportional to the deviation $e_{i}$ is added or subtracted to the modulation law depending on the sign of the deviation,

- the modified modulation law is applied in order to achieve a new time-frequency analysis, and

- the process is iterated until the overall deviation is below a desired threshold.

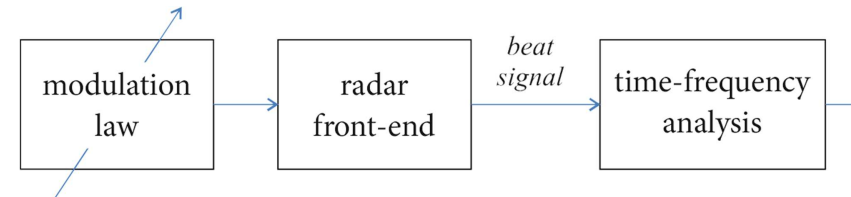

Figure 11: Principle of frequency linearization based on time-frequency analysis of the beat signal.

A Short-Time Fourier Transform (STFT) can be used as a time-frequency analysis to determine the nonlinearities of the beat frequency $f_{b}$. STFT is a Fourier-related transform used to determine the sinusoidal frequency and phase content of local sections of a signal as it changes over time. The result of the frequency linearization process is presented in Figure 12. A Luneburg lens is located about $17 \mathrm{~m}$ from the radar, and a STFT of the beat signal is computed. Figure 12(a) shows the beat signal $s_{b}$ obtained when the initial linear tuning signal is applied, and Figure 12(b) is the result of the STFT: the 
measured beat frequency is not constant over the modulation period $t_{\mathrm{m}}$, with a peak-to-peak variation of about $1.76 \mathrm{kHz}$. The corresponding FFT over the whole beat signal is presented in Figure 12(c). It can be seen that the target cannot be accurately located due to the presence of spurious frequencies, and the range resolution is degraded.

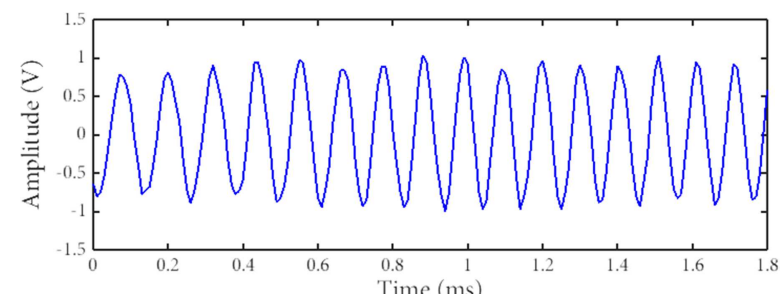

(a)

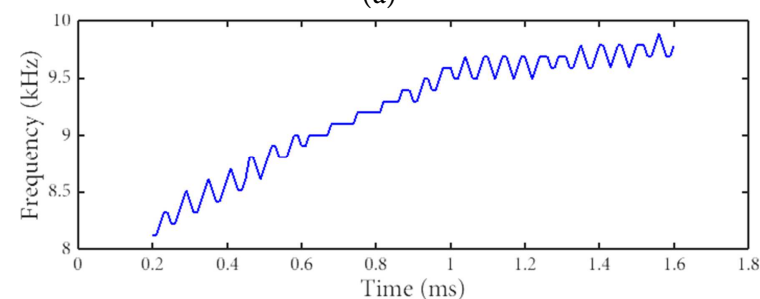

(b)

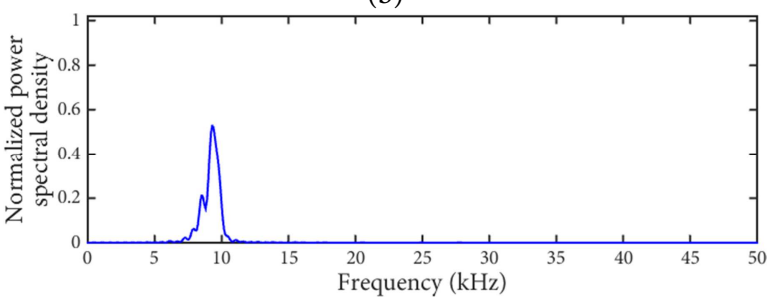

(c)

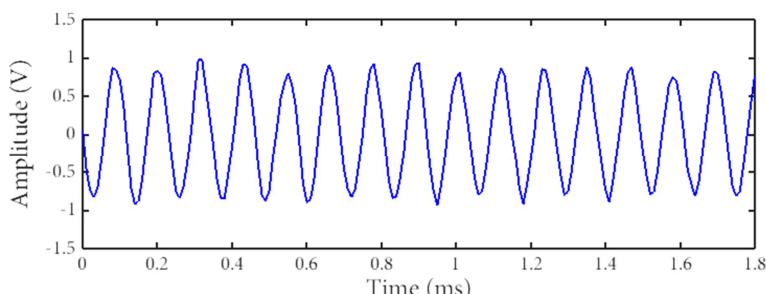

(d)

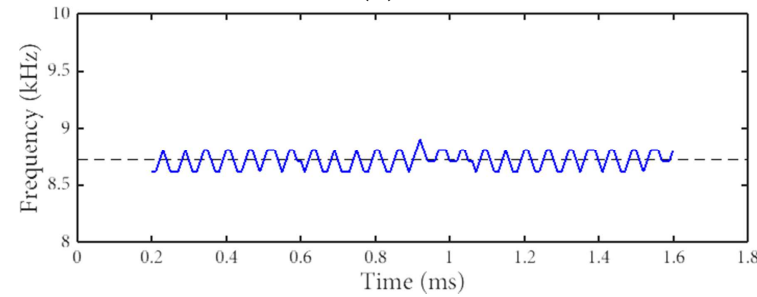

(e)

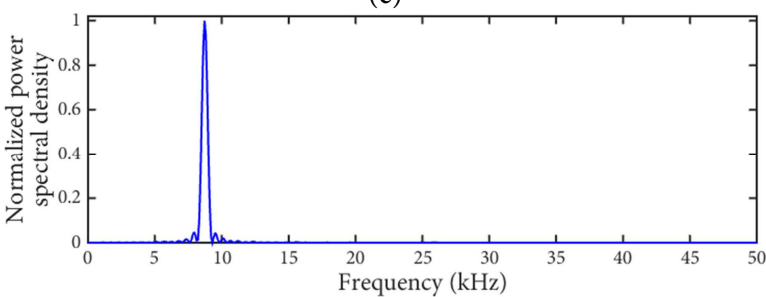

(f)

Figure 12: Frequency linearization. A Luneburg lens is located at range $17 \mathrm{~m}$ in front of the radar, with zero radial velocity. (a) Beat signal with the initial linear modulation law is, and (b) corresponding Short-Time Fourier Transform (STFT). The duration of the sliding window is $0.4 \mathrm{~ms}$ (i.e. 81 signal samples with $f_{\mathrm{s}}=200 \mathrm{kHz}$ ). One can observe that the beat frequency is not constant over the modulation, and the 1024-point FFT of the beat-signal (c) shows the presence of spurious frequencies.

(d) Beat signal with the optimized modulation law, and (e) corresponding STFT. The beat frequency is quite constant over the modulation period. (f) The 1024-point FFT of the beat-signal highlights one unique peak.

The beat signal $s_{\mathrm{b}}$ obtained with the optimized tuning signal is presented in Figure 12(d), and the corresponding STFT in Figure 12(e): the beat frequency is quite constant over the modulation period $t_{\mathrm{m}}$, with a peak-to-peak variation of about $0.29 \mathrm{kHz}$. The FFT over the whole beat signal in Figure 12(f) highlights one unique peak, with a half-power $(-3 \mathrm{~dB})$ width of $497 \mathrm{~Hz}$. This value can be compared with the theoretical expected half-power width of $438 \mathrm{~Hz}$ : the difference of $59 \mathrm{~Hz}$ is introduced by the linearization process which is not perfect, resulting in a larger frequency peak.

The frequency transition at the end of the modulation period $t_{\mathrm{m}}$ (from $f_{0}+\Delta f / 2$ to $f_{0}-\Delta f / 2$ ) introduces disturbances in the measured beat signal as shown in Figure 13(a). The electronic reception stage saturates, and the length of the useful signal can be reduced. To minimize these disturbances, the abrupt frequency transition is replaced with a sinusoidal-like transition. The result is presented in Figure 13(b). In order to maintain the same modulation period $t_{\mathrm{m}}$ (i.e. the same number of radar acquisition per antenna revolution) the slope of the frequency variation is increased, which leads to an increase of the bandwidth of the beat signal. 


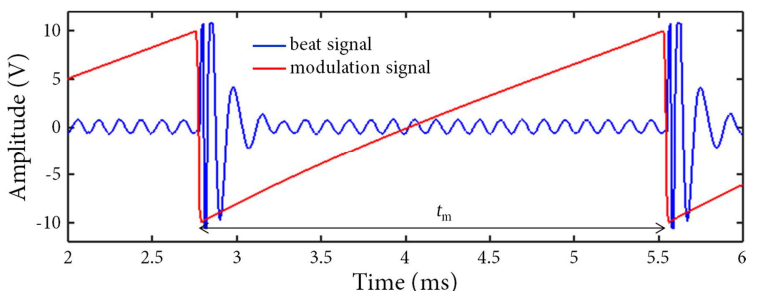

(a)

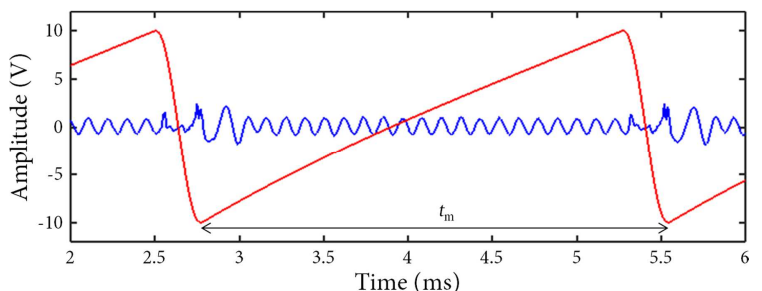

(b)

Figure 13: Influence of the modulation transition. (a) An abrupt frequency transition from $\left(f_{0}+\Delta f / 2\right)$ to $\left(f_{0}-\Delta f / 2\right)$ at the end of the modulation introduces disturbances in the measured beat signal due to saturation effects. (b) A sinusoidal-like transition allows reducing these disturbances, at the expense of an increase of the bandwidth of the beat signal.

Finally, a distance calibration is realized. A point target (Luneburg lens) is positioned in front of the radar, at a range between $5 \mathrm{~m}$ and $100 \mathrm{~m}$. For each reflector position, a radar signal is measured, a radar spectrum is computed and the value of the beat frequency is extracted from the spectrum (position of the maximum of the peak that can be observed in the radar spectrum). The result obtained is presented in Figure 14. The beat frequency vs. distance function highlights a linear behavior, with a coefficient of determination $\mathrm{R}^{2}=1$.

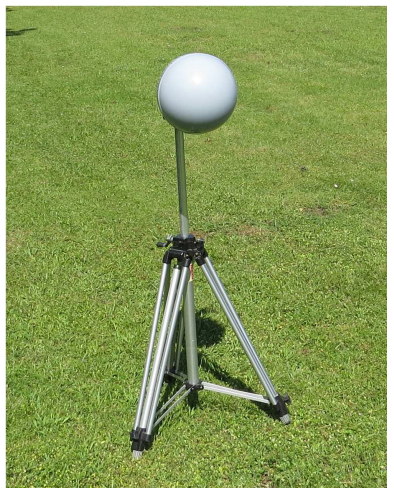

(a)

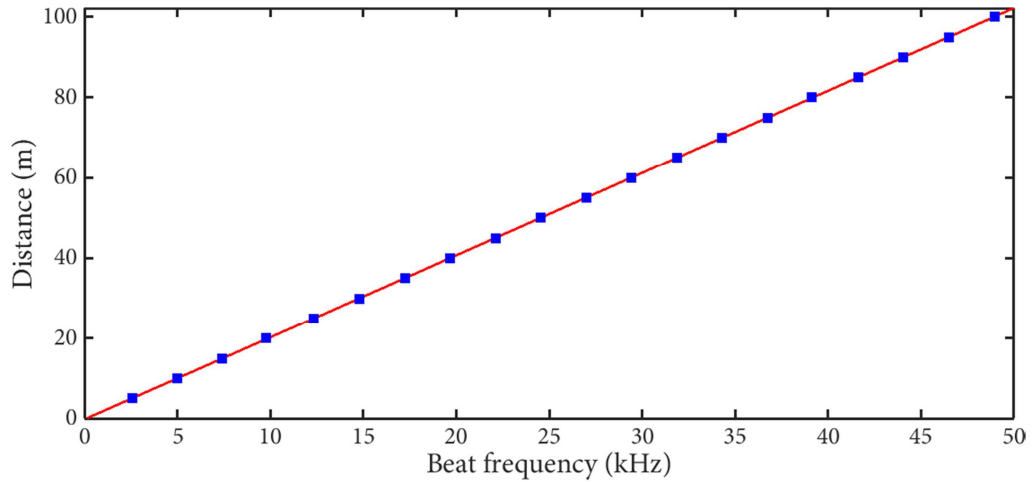

(b)

Figure 14: Beat frequency versus distance function. (a) A point target (Luneburg lens) is placed in front of the radar, at a range between $5 \mathrm{~m}$ and $100 \mathrm{~m}$. (b) For each position, the radar-reflector distance $r$ and the corresponding beat frequency $f_{0}$ are measured (squares). The linear regression highlights a coefficient of determination $\mathrm{R}^{2}=1$.

\subsection{Reception and Data Processing}

A schematic block diagram of the radar electronic reception stage and of the radar processing is presented in Figure 15.

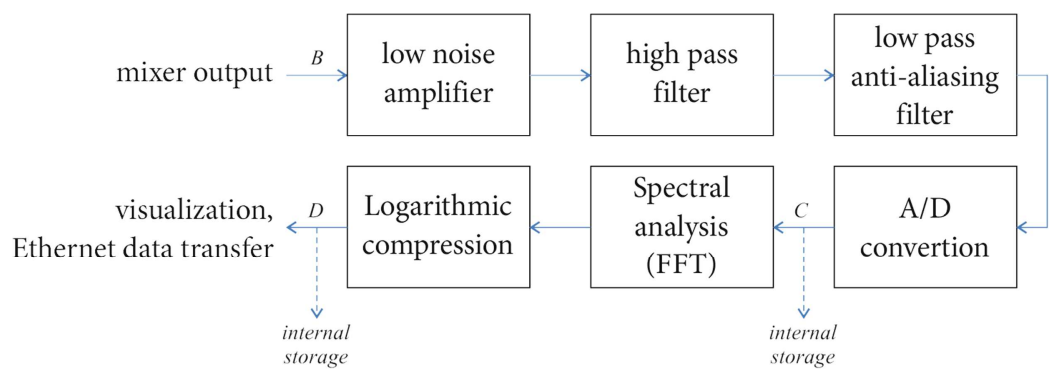

Figure 15: Schematic block diagram of radar signal reception and processing.

The objective of the electronic reception stage is to amplify the radar signal in order to obtain a digitizable signal, and to filter the unwanted frequencies.

Due to the low level of the useful beat signal at the output of the mixer (Figure 15, mark $B$ ), the first reception stage is a low noise amplifier, with a low noise factor and a small amplification gain. 
The high pass filter is a second order filter, which allow compensating the attenuation introduced by the term $r^{-2}$ present in Eq. (7): a point target is detected with the same amplitude independently of the range.

The objective of the analog anti-aliasing filter is to eliminate aliasing, i.e. eliminate the frequency components introduced by targets beyond the maximum distance specified by the sampling rate $f_{\mathrm{s}}$. The design of an efficient analog anti-aliasing filter, which preserves frequencies below $f_{\mathrm{s}} / 2$ and cuts all undesired frequencies above $f_{\mathrm{s}} / 2$, is still a challenge. For that reason we are using an oversampling analog to digital (A/D) converter to digitalize the radar signal. It is associated with a decimation processing (digital low pass filter + downsampling) and it facilitates the design of the analog antialiasing filter. Indeed, the higher sampling rate (versus the sampling rate $f_{\mathrm{s}} / 2$ suggested by the Shannon-Nyquist criterion) significantly eases the transition band requirements of the analog antialiasing filter, and leads to the design of a simple analog low pass filter which rejects high-frequency components before $\mathrm{A} / \mathrm{D}$ conversion.

As beat frequencies $f_{b}$ are proportional to radar-target distances $r$, a spectral analysis is applied on signals after A/D conversion (Figure 15, mark $C$ ). The Fast Fourier Transform (FFT) is the basic signal processing tools for spectral analysis, and it provides real-time and robust spectral analysis even in presence of noise. Typically, we compute a 1024-point FFT of the digitalized beat signal. Other spectral analysis methods such as parametric methods (MUSIC, ESPRIT, etc.) (Schmidt, 1986; Shahbazpanahi, Valaee, \& Bastani, 2001) can be used if higher frequency resolutions are required. These methods are generally very time-consuming and they cannot be computed in real time.

The $r^{-4}$ factor in Eq. (6) introduces a large variation of the received power (for example, a variation of $52 \mathrm{~dB}$ for the same target located at ranges $5 \mathrm{~m}$ and $100 \mathrm{~m}$ ), but this variation is electronically compensated. However, the RCS $\sigma$ of a target can span several orders of magnitude, and the corresponding received power $p_{\mathrm{r}}$ highlights the same dynamic range. The logarithmic compression allows reducing this dynamic range, and the received power is represented in $\mathrm{dB}$

$$
p_{\text {rlog }}=10 \log _{10}\left(p_{\mathrm{r}}\right) \text {. }
$$

Finally, several information are acquired simultaneously with the beat signal:

- for each beat signal measurement, the angular position of the antenna is measured. Data from the antenna encoder are synchronized with the modulation signal. Thus, the acquisition rate of the antenna encoder is $f_{\mathrm{m}}=360 \mathrm{~Hz}$.

- The transition $360^{\circ} \rightarrow 0^{\circ}$ of the antenna encoder activates an electronic signal which is used to synchronize the acquisition of data coming from the proprioceptive sensors (gyrometer and odometer), and from the GPS (if present). As the rotation velocity of the antenna is $\omega=60 \mathrm{rpm}=2 \pi \mathrm{rad} / \mathrm{s}$, the acquisition rate is $1 \mathrm{~Hz}$.

Raw data (Figure 15, mark $C$ ) or processed data (Figure 15, mark $D$ ) can be stored internally on a flash disk, and/or transmitted for visualization and further treatment using an Ethernet link. Data recorded during one antenna turn typically includes:

- 360 radar signal of $N$ samples

or

360 radar spectra of $M$ points, with $M=$ (number of FFT points) / 2 ,

- 1 odometer information (distance travelled by the radar during the antenna revolution),

- 1 gyrometer information (angular rotation of the radar during the antenna revolution),

- 1 GPS data sentence (GPS position at the beginning of the antenna revolution). 


\section{Radar Spectrum and 2D Image Construction}

An example of radar spectrum is presented in Figure 16. Figure 16(a) is an aerial image of the test zone, extracted from Google Earth data (localization: $\left.45^{\circ} 45^{\prime} 45.86^{\prime \prime} \mathrm{N}, 3^{\circ} 06^{\prime} 35.66^{\prime \prime} \mathrm{E}\right)$. The dash-dotted line indicates the line of sight of the radar, and the solid lines represent the $-3 \mathrm{~dB}$ aperture of the antenna. Several elements are present in the radar field of view: trees (marks $A, B$ and $D$ ), Luneburg lens (represented with a circle, mark $C$, see Figure 14(a)) and building (marks $E$ and $F$ ). Figure 16(b) is the corresponding radar spectrum obtained with a 1024-point FFT and a rectangular window function. Trees, building and lens are detected with specific frequency peaks. Two vertical steel beams integrated in the structure of the building are localized with marks $E$ and $F$ in Figure 16(a). These steel beams create metallic corner reflectors that are clearly detected by the radar.

Strong sidelobes (spectral leakage), due to the use of the rectangular window function, can be observed on both sides of the frequency peaks. These sidelobes can be reduced with the use of a window function other than the rectangular one. Result obtained with a Hann window function is presented in Figure 16(c). The amplitudes of sidelobes are reduced, but at the cost of larger main lobes, and therefore a lower distance resolution.

The echo from the Luneburg lens (mark $C$ ) allows measuring experimentally the distance resolution $\delta r$. The distance resolution $\delta r$, as describe in Eq. (8), corresponds to the Rayleigh criterion and it can be measured from the curve in Figure 16(b): it is the half of the gap between the first minimums around the central maximum. From Figure 16(b), we obtain a distance resolution $\delta r \approx 1.04 \mathrm{~m}$, which is coherent with the theoretical value $1.05 \mathrm{~m}$ obtained with Eq. (8) in Section 3.1.2. In radar domain, the half power $(-3 \mathrm{~dB})$ distance resolution $\delta r_{-3 \mathrm{~dB}}$ is commonly used: it is the peak width at half of its height. This criterion is less restrictive than the Rayleigh criterion. From Figure 16(b), we obtain a half power distance resolution $\delta r_{-3 \mathrm{~dB}}=0.91 \mathrm{~m}$. The final $-3 \mathrm{~dB}$ distance resolution measured with the echo from the Luneburg lens and a Hann window function (Figure 16(c)) is $\delta r_{-3 \mathrm{~dB}}=1.48 \mathrm{~m}$.

The principle of panoramic image construction is shown in Figure 17. Figure 17(a) is an aerial image of the test zone, extracted from Google Earth data (localization: $45^{\circ} 45^{\prime} 45.86^{\prime \prime} \mathrm{N}, 3^{\circ} 06^{\prime} 35.66^{\prime \prime} \mathrm{E}$ ). The dash-dotted lines indicate the initial and final lines of sight. The image is constructed progressively, with the juxtaposition of the successive radar spectra. A 3D representation with 26 radar spectra is shown in Figure 17(b). The Plan Position Indicator (PPI) image is a top-view 2D representation of the surrounding environment over $360^{\circ}$. The distance between an element of the image and the center of the image indicates the range, and the angle around the display is the azimuth. A sector of PPI image is presented in Figure 17(c). The color level indicates the amplitude of the backscattered radar signal.

It must be remembered that PELICAN antenna is a fan-beam antenna, with a large aperture in the vertical dimension. With such an antenna, the altitudes or heights of the detected elements cannot be determined, and the echoes are projected onto the rotation plane of the antenna. The main consequence is that PELICAN radar can only build 2D images of the environment. 
(a)

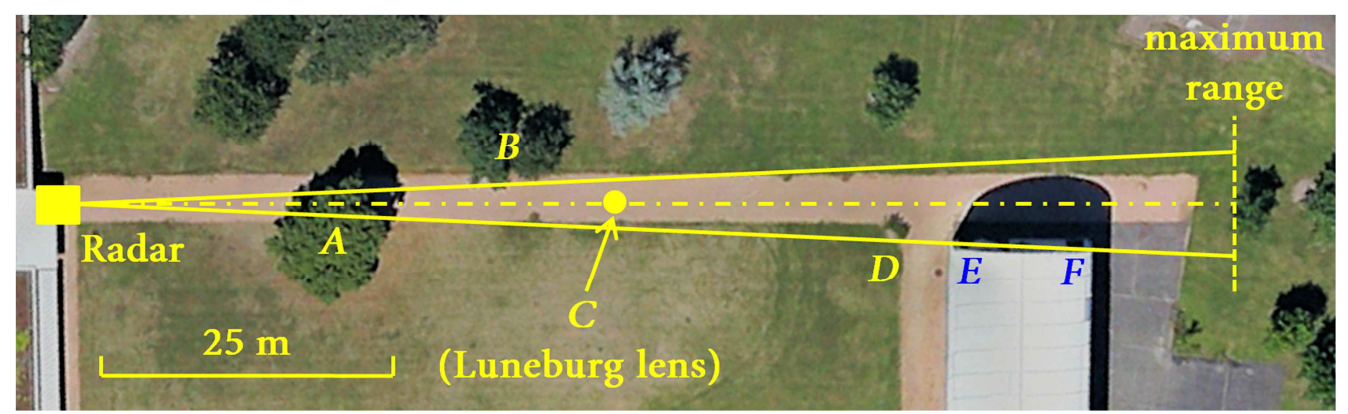

(b)

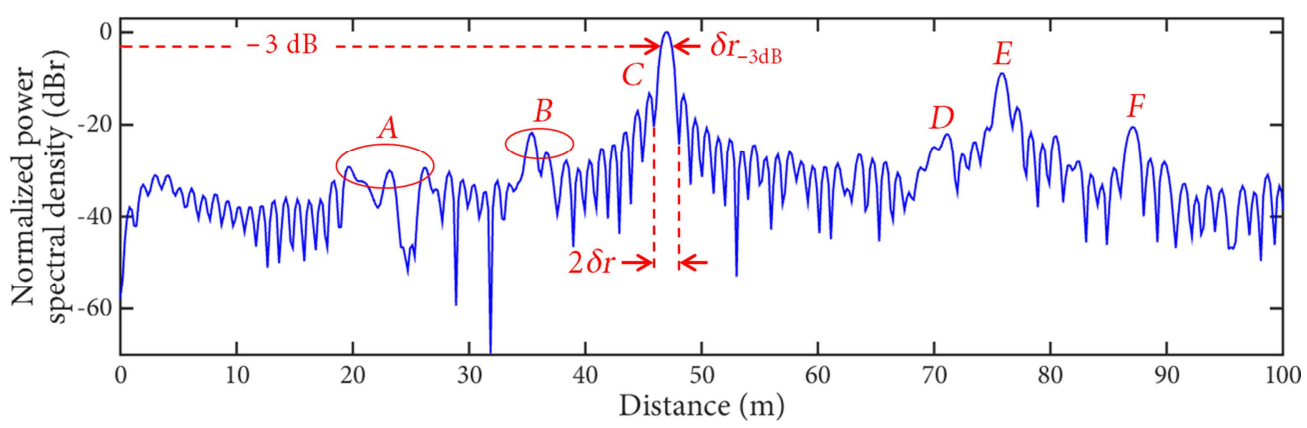

(c)

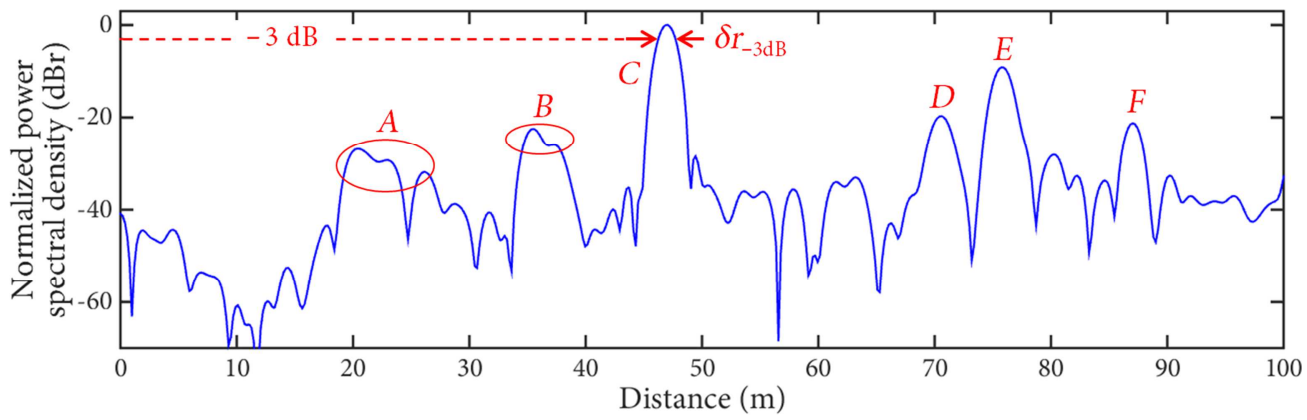

Figure 16: Example of radar spectrum. (a) Aerial image of the test zone. The dash-dotted line indicates the line of sight of the radar, and the solid lines the $-3 \mathrm{~dB}$ aperture of the antenna. Several targets are present: trees (marks $A, B$ and $D$ ), a Luneburg lens (mark $C$ ) and a building (marks $E$ and $F$ ). (b) Radar spectrum computed with a rectangular window function. (c) Radar spectrum computed with a Hann window function. 


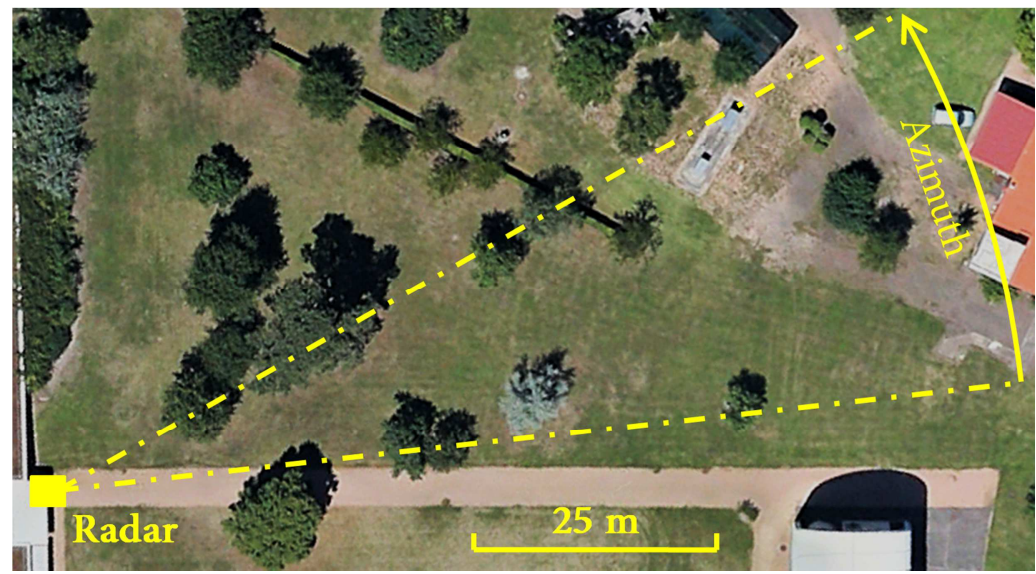

(b)

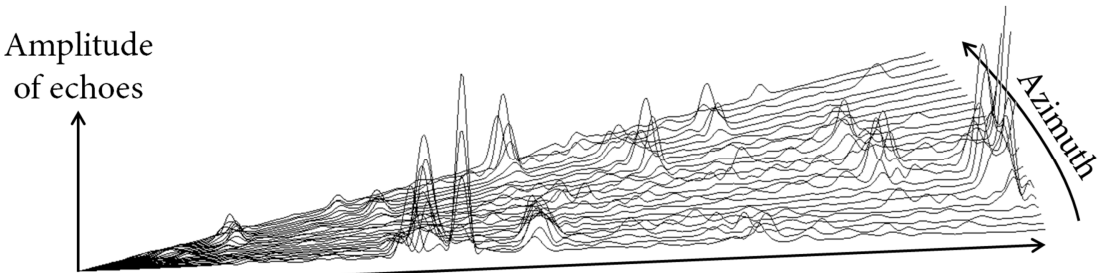

Distance

(c)

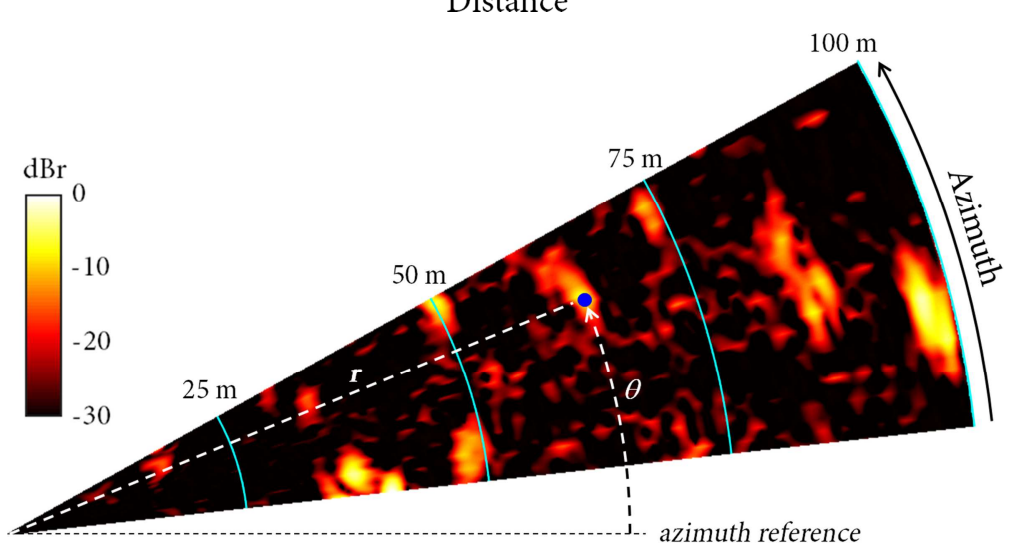

Figure 17: Example of radar image construction. (a) Aerial image of the test zone. The dash-dotted lines indicate the initial and final lines of sight. (b) The image is constructed gradually with the successive radar spectra (one spectrum at each degree of antenna rotation). (c) A PPI radar image is a top-view of the successive radar spectra. A target is detected with $(\theta, r)$ polar coordinates.

PELICAN radar provides raw data in polar coordinates (azimuth $\theta$, distance $r$ ). Examples of polar images of a point target (Luneburg lens) located at range $13 \mathrm{~m}$ and $64 \mathrm{~m}$ are presented in Figure 18(a) and Figure 19(a) respectively. Each column of the figures corresponds to a single radar spectrum (i.e. one antenna pointing direction). Also called B-Scope, the polar image allows plotting the power received from the targets without distortion. It can be seen in both images that the echoes from the point targets are similar, and are independent of the range (the azimuth and distance scales are identical for ease of images comparison).

Generally, polar coordinates $(\theta, r)$ are transformed to Cartesian coordinates $(x, y)$. Cartesian coordinates facilitate the visualization of radar images. They are also relevant for image processing and navigation algorithms, because data used by these algorithms (measurements, estimates, etc.) are mainly based on Cartesian coordinates. In that case, the values of received power and the corresponding coordinates must be converted to the Cartesian system of coordinates, but this transformation can be source of confusion. Polar images of the point target in Figure 18(a) (distance $13 \mathrm{~m}$ ) and Figure 19(a) (distance $64 \mathrm{~m}$ ) are converted to Cartesian images in Figure 18(b) and Figure 19(b) respectively (the $X$ and $Y$ distance scales are identical for ease of images comparison). 


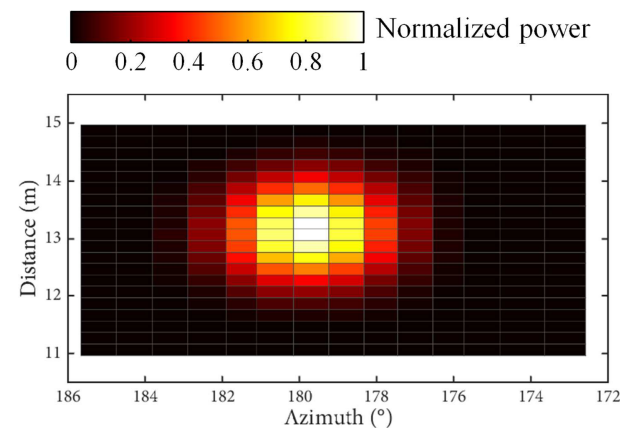

(a)

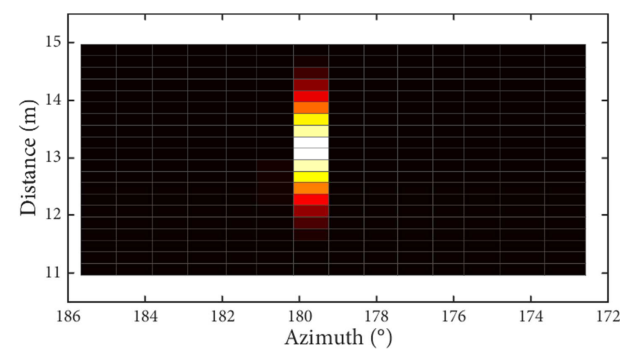

(c)

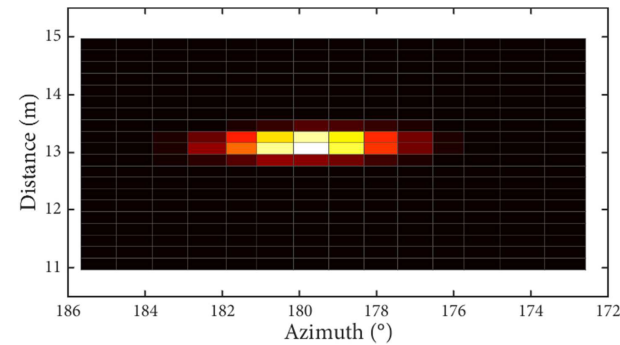

(e)

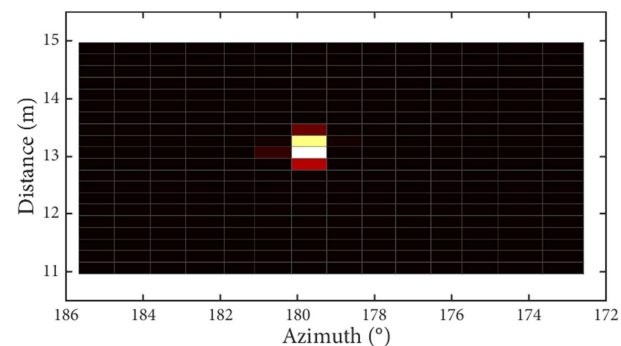

(g)

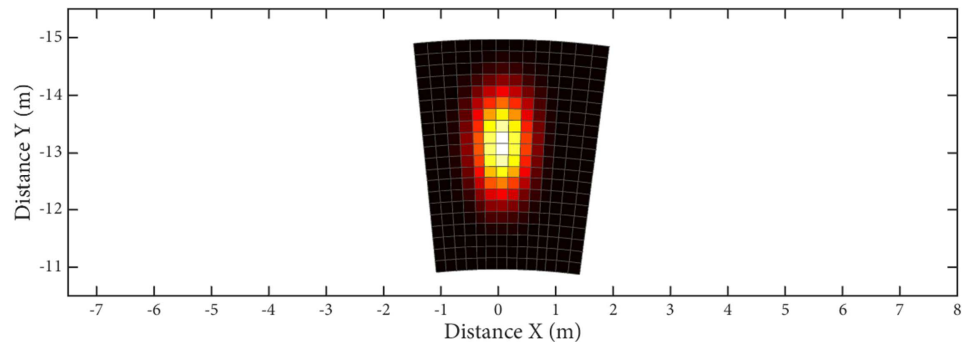

(b)

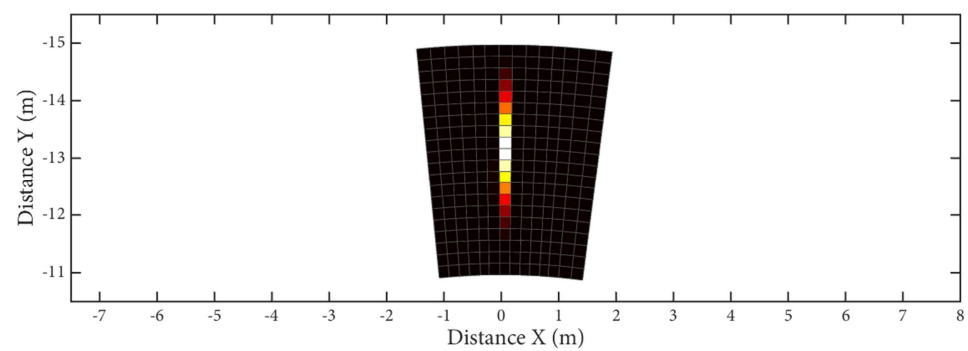

(d)

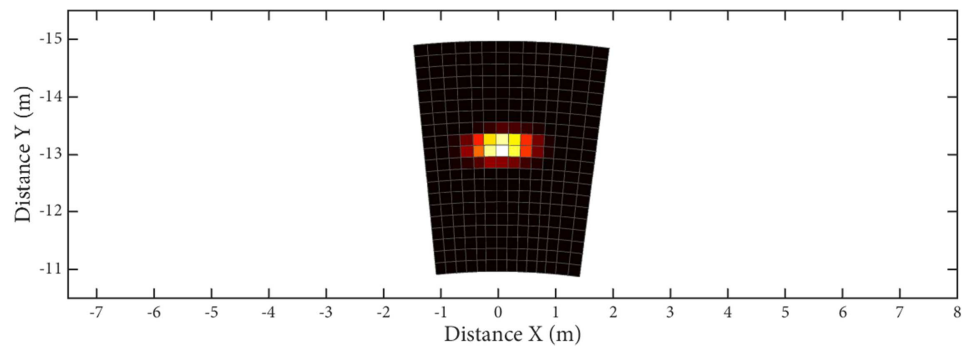

(f)

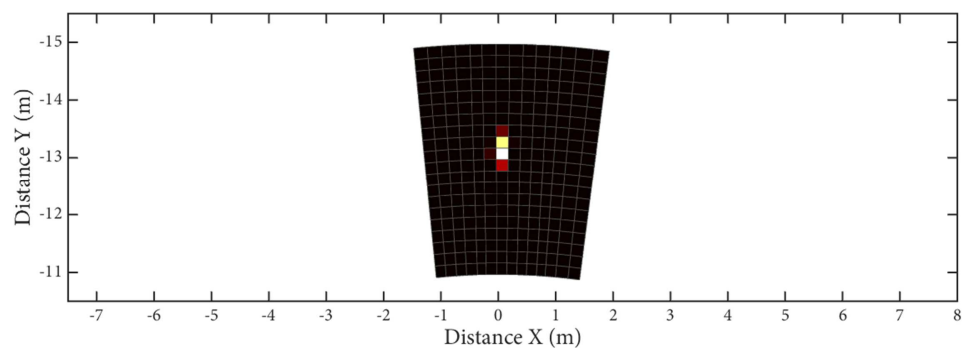

(h)

Figure 18: Radar image of a point target (Luneburg lens) located at range $13 \mathrm{~m}$. (a) and (b): raw radar images in polar and Cartesian coordinates respectively. (c) and (d): Richardson-Lucy deconvolution over the azimuth dimension, in polar and Cartesian coordinates respectively. (e) and (f): Richardson-Lucy deconvolution over the range dimension, in polar and Cartesian coordinates respectively. (g) and (h): 2D Richardson-Lucy deconvolution, in polar and Cartesian coordinates respectively.

It can be seen in both images that the echoes width over the $Y$-axis (which corresponds to the distance dimension of the polar image in this example) is similar: the half power width is about $1.47 \mathrm{~m}$. But the echoes width over the $X$-axis (which corresponds to the azimuth dimension of the polar image in this example) differs: the half power width is about $0.92 \mathrm{~m}$ at range $13 \mathrm{~m}$ and $4.5 \mathrm{~m}$ at range $64 \mathrm{~m}$. This phenomenon is an intrinsic deformation of Cartesian images, and it is introduced by the antenna beamwidth. A given target has the same angular occupancy independent of the range in polar coordinates; but this target will have different spatial occupancies in Cartesian coordinates, proportionately to range and antenna beamwidth. 


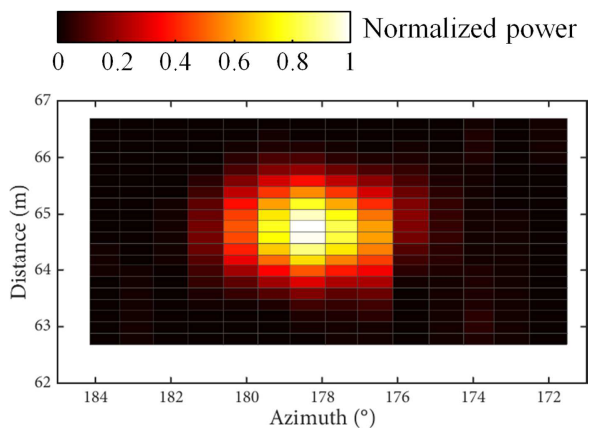

(a)

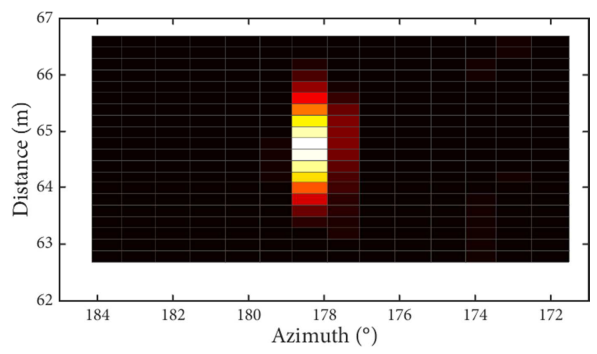

(c)

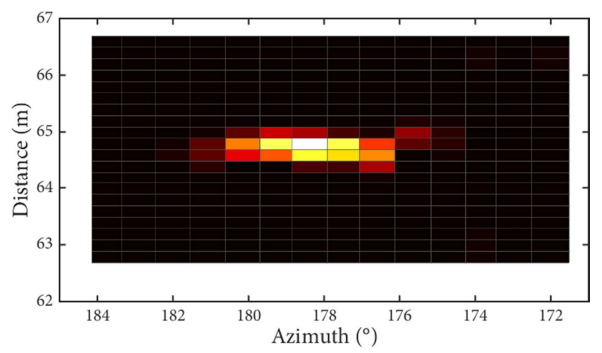

(e)

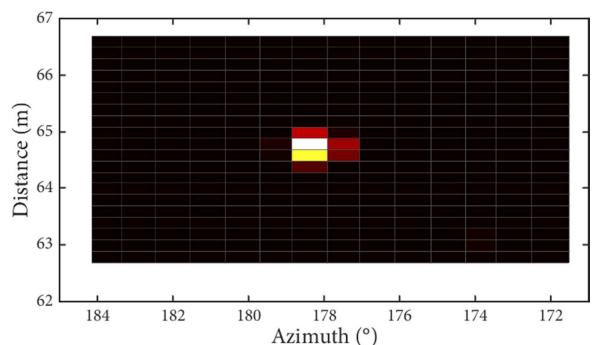

(g)

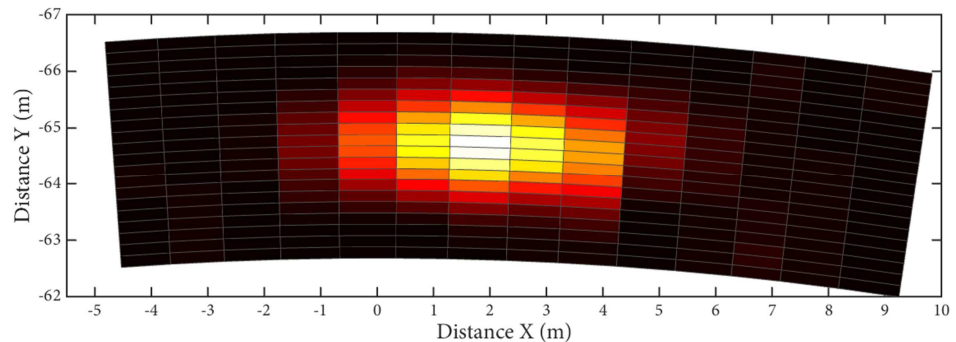

(b)

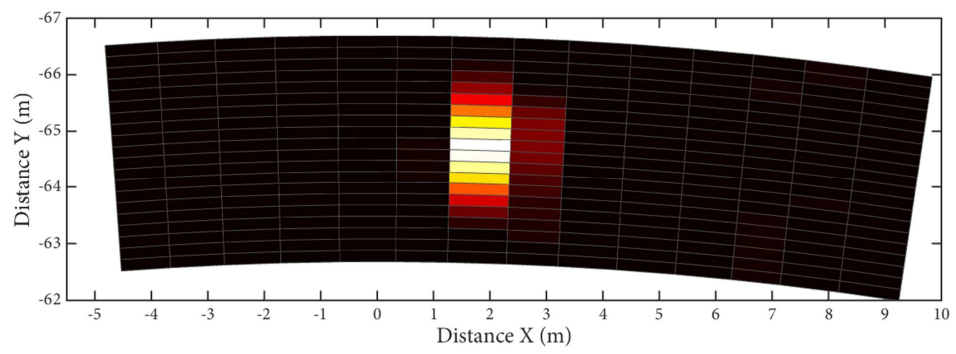

(d)

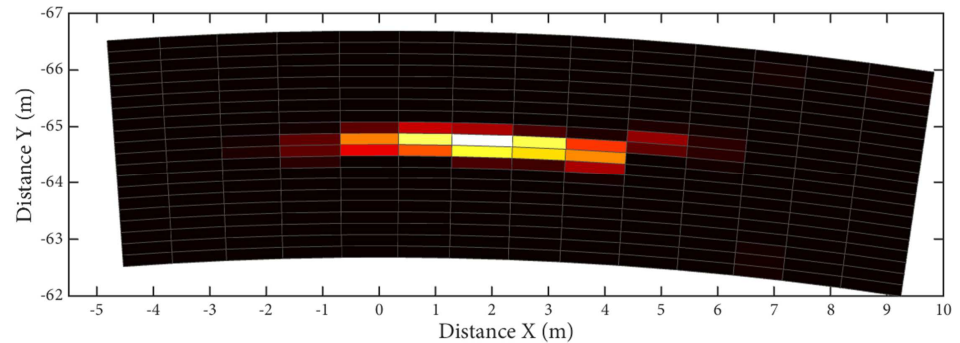

(f)

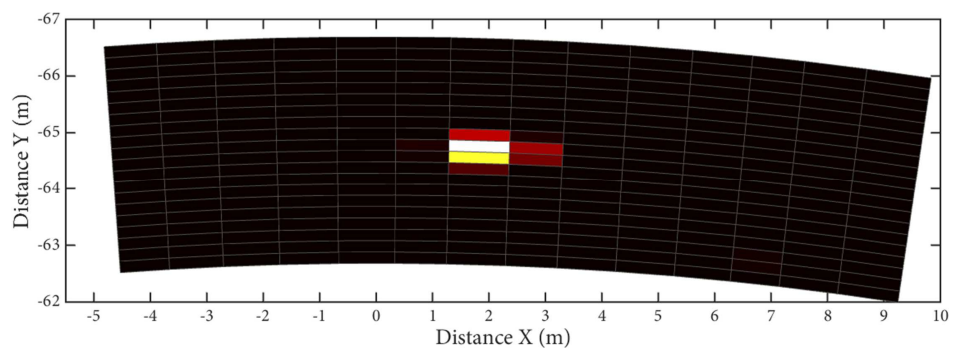

(h)

Figure 19: Radar image of a point target (Luneburg lens) located at range $64 \mathrm{~m}$. (a) and (b): raw radar images in polar and Cartesian coordinates respectively. (c) and (d): Richardson-Lucy deconvolution over the azimuth dimension, in polar and Cartesian coordinates respectively. (e) and (f): Richardson-Lucy deconvolution over the range dimension, in polar and Cartesian coordinates respectively. (g) and (h): 2D Richardson-Lucy deconvolution, in polar and Cartesian coordinates respectively.

\section{Resolution Improvement}

\subsection{Angular Resolution}

The angular resolution $\delta_{\Theta}$ is defined as the minimum angular separation for which two equal point targets can be resolved when located at the same range. This angular resolution is determined by the aperture of the antenna beam. A rough estimation of the antenna half power beamwidth $\Theta$ (expressed in radians) is given by the ratio of the wavelength $\lambda$ to the antenna size $d$ (Nathanson, 1999) 


$$
\Theta=\delta_{\Theta}=\frac{\lambda}{d} .
$$

According to Eq. (20), we obtain an angular resolution of $4.5^{\circ}$ in the azimuth plane with the characteristics of PELICAN radar (i.e. $\lambda=1.25 \mathrm{~cm}$ and $d=15.8 \mathrm{~cm}$ ). This value is not far from the value of $5^{\circ}$ provided by the manufacturer (see Table 3 ). The effective half power can be measured with data used in Figure 18(a) or Figure 19(a): we obtain an angular resolution of about $4^{\circ}$, which is a value slightly better than the value announced by the manufacturer.

It could be interesting to use an antenna with a smaller beamwidth $\Theta$ (i.e. a better angular resolution $\delta_{\Theta}$ ), particularly when considering the deformations introduced by the polar to Cartesian transformation (see Section 4). From Eq. (20), it comes that a better angular resolution can be obtained with (i) a decrease of the wavelength $\lambda$, and/or (ii) an increase of the dimension $d$ of the antenna. But due to external criteria (bandwidth limitations, maximum size of the antenna, etc.), neither the wavelength $\lambda$ nor the dimension $d$ can be modified, so another solution has to be found.

In aerial or satellite radar remote sensing, Synthetic Aperture Radar (SAR) is widely used (Ford et al., 1993; Ferretti, Monti-Guarnieri, Prati, Rocca, \& Massonnet, 2007). Because of the radar-target distances considered in airborne or spaceborne applications, the antenna aperture leads to a wide radar footprint and a poor angular resolution in the azimuth plane. But the problem is that the antenna size cannot be increased at will in order to reduce the antenna aperture. SAR works like a phased-array antenna, using one antenna in a time-multiplex process: the successive positions of the antenna are used as the element of an extremely large antenna which produces a narrow beamwidth. But SAR signal processing implies an exact knowledge of the path and velocity of the platform, which is by itself a non-trivial problem when considering a robot following an unknown trajectory in an unknown and non-flat environment.

The proposed solution considers that the detection of a target with a scanning radar can be seen as the convolution of the target with the antenna beam. Such an approach is used in astronomy, where light from the stars is deformed (convolved) by the geometry of the optical lenses. The distortion introduced by the imaging instrument (telescope, radar, etc.) can be expressed as

$$
g=f \otimes h+n,
$$

where $g$ is the acquired image, $f$ the real image (expected to be recovered), $n$ an additive noise, $h$ the Point Spread Function (PSF) of the imaging system, and $\otimes$ the convolution operator. The PSF describes the response of the imaging system to a point target, i.e. how the signal from a point target is spread by the imaging system. Several deconvolution algorithms are proposed in the literature to restore the original image $f$. These algorithms can be non-iterative algorithms (regularized inversefiltering algorithm (Preza, Miller, Thomas, \& McNally, 1992), Wiener filtering algorithm (Shaw \& Rawlins, 1991), etc.), or iterative algorithms such as Carrington algorithm (Carrington et al., 1995) or Richardson-Lucy algorithm (Richardson, 1972; Lucy, 1974).

The Richardson-Lucy algorithm is a well-known Bayesian-based method for the deconvolution of images convolved with a known PSF. Well adapted to the localization of point sources, it is commonly used in astronomy (it is known to be used for the Hubble Space Telescope). It has also been used for the deconvolution of radar images (Diewald, Klappstein, Dickmann, \& Dietmayer, 2011; Zha, Zhang, Huang, \& Yang, 2015). The Richardson-Lucy algorithm follows the next iterative procedure

$$
f_{k+1}=\left(h * \frac{g}{f_{k} \otimes h}\right) f_{k},
$$


where $f_{k}$ denotes the reconstructed image at the $k$-th iteration, $f_{k+1}$ the next iteration of the reconstructed image, and $*$ is the correlation coefficient operator.

The accuracy of the Richardson-Lucy deconvolution is closely related to the quality of the PSF. In our case, the PSF is related to the radiation pattern of the antenna in the azimuth plane as shown in Figure 8 . The radiation pattern provided by the manufacturer could be used as PSF, but data accuracy appears to be not sufficient. As the PSF is the response of the radar system to a point target, it can be estimated with radar measurements. Such a measurement is presented in Figure 20, with a point target (Luneburg lens) located in front of the radar. In this example, the radar-target distance is $17 \mathrm{~m}$, but the distance is without important. The radar measurements (circles) are approximated with a Gaussian curve, and this curve is used as PSF in the deconvolution process.

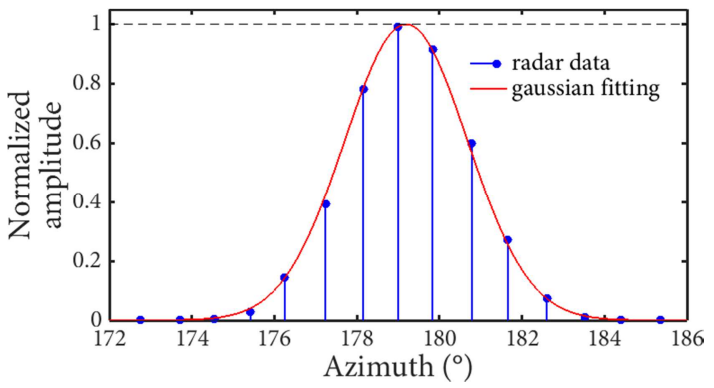

Figure 20: Point spread function for the Richardson-Lucy deconvolution over the azimuth axis. A point target (Luneburg lens) is located in front of PELICAN radar. Radar measurements (circles) are approximated with a Gaussian curve which is used as PSF.

Examples of Richardson-Lucy deconvolution over the azimuth axis obtained with this PSF are presented in Figure 18 (radar-target distance $13 \mathrm{~m}$ ) and Figure 19 (radar-target distance $64 \mathrm{~m}$ ): polar coordinates in Figure 18(c) and Figure 19(c); Cartesian coordinates in Figure 18(d) and Figure 19(d). The number of iterations is 20 in these examples. The result of the Richardson-Lucy deconvolution can be compared with the raw radar images in Figure 18(a,b) and Figure 19(a,b). One can observe that the angular resolution is improved, resulting in a smaller spatial occupancy in Cartesian coordinates.

\subsection{Distance Resolution}

When using the Fourier transform for frequency analysis, it can be seen from Eq. (8) that the range resolution $\delta r$ only depends on the sweep frequency $\Delta f$ an increase of the sweep frequency allows obtaining a better distance resolution. But due to technological limits or regulatory constraints, the value $\Delta f$ cannot be increased at will. And if we consider PELICAN radar, $\Delta f$ is set at $195 \mathrm{MHz}$ and this value cannot be increased.

If the Fourier transform is the basic tool for spectrum analysis due to its robustness, its noise resistance, and its efficient software implementation (FFT algorithm), it exists numerous signal processing methods for high resolution spectrum estimation: Yule-Walker, Prony, MUSIC, ESPRIT, etc. (Schmidt, 1986; Shahbazpanahi et al. 2001; Shanker \& Arai, 2003; Abou-Khousa, Simms, Kharkovsky,\& Zoughi, 2009; Bency, Lal, \& Abraham, 2013). These methods take advantage of known parameters of the signal such as the number of spectral components. We have developed such an approach with the use of ESPRIT (Estimation of Signal Parameter via Rotational Invariance Technique) algorithm in Rouveure, Faure, Jaud, Monod, \& Moiroux-Arvis (2014). The major limitations concern the sensitivity to signal to noise ratio, and long computation times. For these reasons, we have studied an approach based on deconvolution. If we observe the echo from the point target in Figure 16(b) (mark $C$ ), this echo can be seen as the convolution between an impulse function and the cardinal sine function (sinc) introduced by the calculation of the Fourier Transform. The developed idea is to use the sinc function as PSF within a Richardson-Lucy deconvolution process. The 
PSF used is presented in Figure 21, considering a Hann window function for the calculation of the 1024-point FFT.

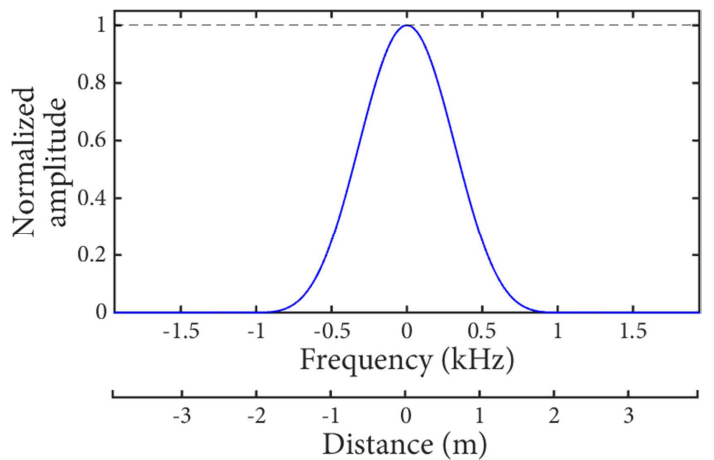

Figure 21: Point spread function for the Richardson-Lucy deconvolution over the distance axis.

Examples of Richardson-Lucy deconvolution over the distance axis obtained with this PSF are presented in Figure 18 (radar-target distance $13 \mathrm{~m}$ ) and Figure 19 (radar-target distance $64 \mathrm{~m}$ ): polar coordinates in Figure 18(e) and Figure 19(e); Cartesian coordinates in Figure 18(f) and Figure 19(f). The number of iterations is 20. The initial raw radar images are shown in Figure 18(a,b) and Figure 19(a,b). The deconvolution process allows reducing the width of the echo over the distance axis.

It must be noticed that this approach allows creating echoes with steep-edged limits. But contrary to high resolution methods, it is not possible to separate frequency components which are not resolved with the Fourier analysis.

And finally, the deconvolutions over the azimuth and distance axis can be combined in order to achieve a 2D deconvolution. Based on the PSF presented in Figure 20 and Figure 21, a 2D PSF can be constructed. This 2D PSF is shown in Figure 22.

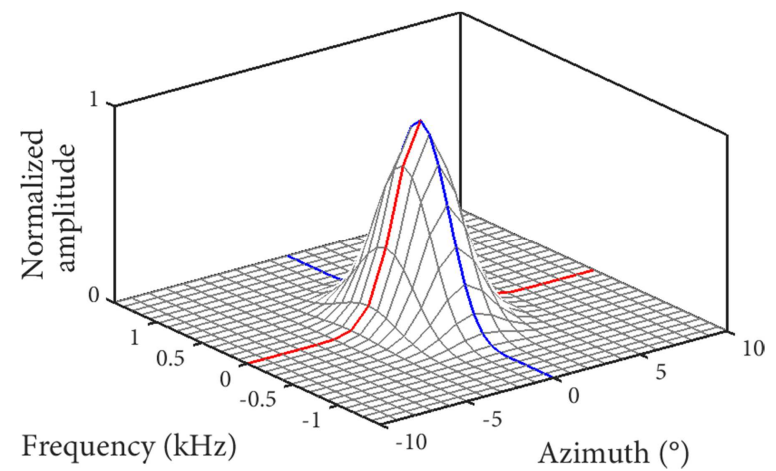

Figure 22: 2D point spread function for the Richardson-Lucy deconvolution. The red and blue lines indicate the PSF over the azimuth and distance axis which are used to build the 2D PSF.

The results of this 2D Richardson-Lucy deconvolution are presented in Figure 18 (radar-target distance $13 \mathrm{~m}$ ) and Figure 19 (radar-target distance $64 \mathrm{~m}$ ): polar coordinates in Figure $18(\mathrm{~g})$ and Figure 19(g); Cartesian coordinates in Figure 18(h) and Figure 19(h). The number of iterations is 20. In comparison to the initial raw images presented in Figure 18(a,b) and Figure 19(a,b), one can observe that the spreads introduced by the antenna beamwidth (azimuth axis) and by the cardinal sine function (distance axis) are strongly reduced. 


\section{Experimental Results in Various Environments}

\subsection{Radar image correction}

PELICAN radar provides each second one panoramic raw image in polar coordinates. As the radar moves during the antenna revolutions, corrections have to be done in order to build consistent panoramic representation of the environment:

- correction of Doppler shift,

- and correction of the distortions introduced by the movement of the robot.

In addition, radar images are subject to Speckle effect. Speckle is a random amplitude modulation which results in a grainy salt-and-pepper pattern in radar images. It is an inherent process to any radar image construction, and it is introduced by phase-interference (fading). A specific filtering procedure has to be applied in order to reduce these effects.

\subsubsection{Correction of Doppler Shift}

It is recalled that PELICAN radar uses a sawtooth modulation. Such a modulation does not allow measuring the radial velocities of the targets. Consequently PELICAN radar assumes a static environment configuration (no mobile targets) and the use of a proprioceptive sensor to measure the velocity of the robot. Equation (3) gives the expression of the Doppler shift $f_{\mathrm{d}}$ introduced by the movement of the robot. $f_{\mathrm{d}}$ depends on the velocity $v$ of the vehicle (measured with a proprioceptive sensor) and on the antenna pointing direction $\theta$ (i.e. the angle between the direction of the vehicle and the antenna direction of propagation). If radar is aligned with the main axis of the robot, $\theta$ is given by the antenna encoder. Once $f_{\mathrm{d}}$ has been computed, each radar spectrum is shifted up or down in order to recover the correct radar-target distances based on Eq. (4).

\subsubsection{Correction of Vehicle Motion}

Because of robot movements during the rotation of the antenna, distortions are introduced between the 360 radar spectra which are used to produce one panoramic radar image. If the movements of the robot are neglected, the targets located around the radar could be detected with deformed shapes and incorrect positions.

In order to take into account the movement of the robot, two proprioceptive sensors are used to estimate the trajectory of the robot during one antenna revolution: an odometer measures the longitudinal displacement; and a gyrometer measures the rotation. The odometer is a radar-based sensor, commonly used with agricultural vehicles. The advantage of this sensor is that it allows measuring the covered distance without slippage even in presence of loose or slippery ground. The gyrometer is a single-axis interferometric fiber-optic system, which highlights excellent resolution and stability. By combining data from the odometer and from the gyrometer with data from the antenna encoder, the 360 radar spectra can be projected in a common reference frame in order to build one consistent revolution radar image.

\subsubsection{Speckle Filtering}

Power reflected from distributed targets can be seen as the sum of all the elementary reflectors or scatterers present in the antenna beam at the same range. From Eq. (1), it appears that the measured radar signal is a coherent sum: owing to the phase term $\Phi_{i}$ of the beat signal $s_{b}$, the sum highlights constructive and destructive interferences, i.e. radar signal is subject to fading effects (Mott, 2007; Argenti, Lapini, Bianchi, \& Alparone, 2013). Under the assumptions that (i) the number of scatterers is large and scatterers are statistically identical and independent, and (ii) no single scatterer has a crosssection significantly larger than the average of all scatterers, the received power for each range bin will 
vary in a random fashion. When considering radar of imaging type, these random amplitude variations produce a speckle pattern, which is a manifestation of fading statistics. The mean amplitude of the Speckle noise can span several orders of magnitude, depending on the material of the targets, and on the angle of the incident wave.

As PELICAN radar is positioned on a moving platform, successive panoramic radar images of almost the same area are available and a specific multi-look filter has been developed to reduce the effect of speckle noise. This speckle noise reduction is obtained through a sliding non-coherent average of $P$ panoramic images. In order to compute the average, the $P$ panoramic images are projected onto a common space through a sliding dead reckoning phase: a gyrometer and an odometer measure the angular variations and the longitudinal displacements between images, in order to compute their relative positions and orientations.

Soil clutter is an illustration of Speckle effect. Features such as soil, grass, etc., represent rough surfaces for the radar (the roughness of a surface is defined relatively to the wavelength of the incident wave), comprising an important number of elementary reflectors. Signals backscattered from all these reflectors have random phases, and they will interfere both constructively and destructively. An example of soil clutter and Speckle effect obtained with PELICAN radar is presented in Figure 23. An aerial image of the test area, extracted from Goggle Earth data (localization: 45 $42^{\prime} 59.90^{\prime \prime} \mathrm{N}$, $\left.3^{\circ} 06^{\prime} 18.80^{\prime \prime} \mathrm{E}\right)$, is presented in Figure 23(a). This area includes a flat grassy area, a vegetation area (trees) and a paved road, labelled $A, B$ and $C$ respectively. The cross indicates the radar position. The raw radar image in Cartesian coordinates is shown in Figure 23(b). Radar spectra are computed with a 1024-point FFT and a Hann window function. The Speckle effect can be observed through the amplitude variations over the flat grassy surface or the vegetation area. The vehicle moves with a velocity of about $4.5 \mathrm{~m} / \mathrm{s}$, and 10 successive raw radar images are used to produce the final image presented in Figure 23(c). 


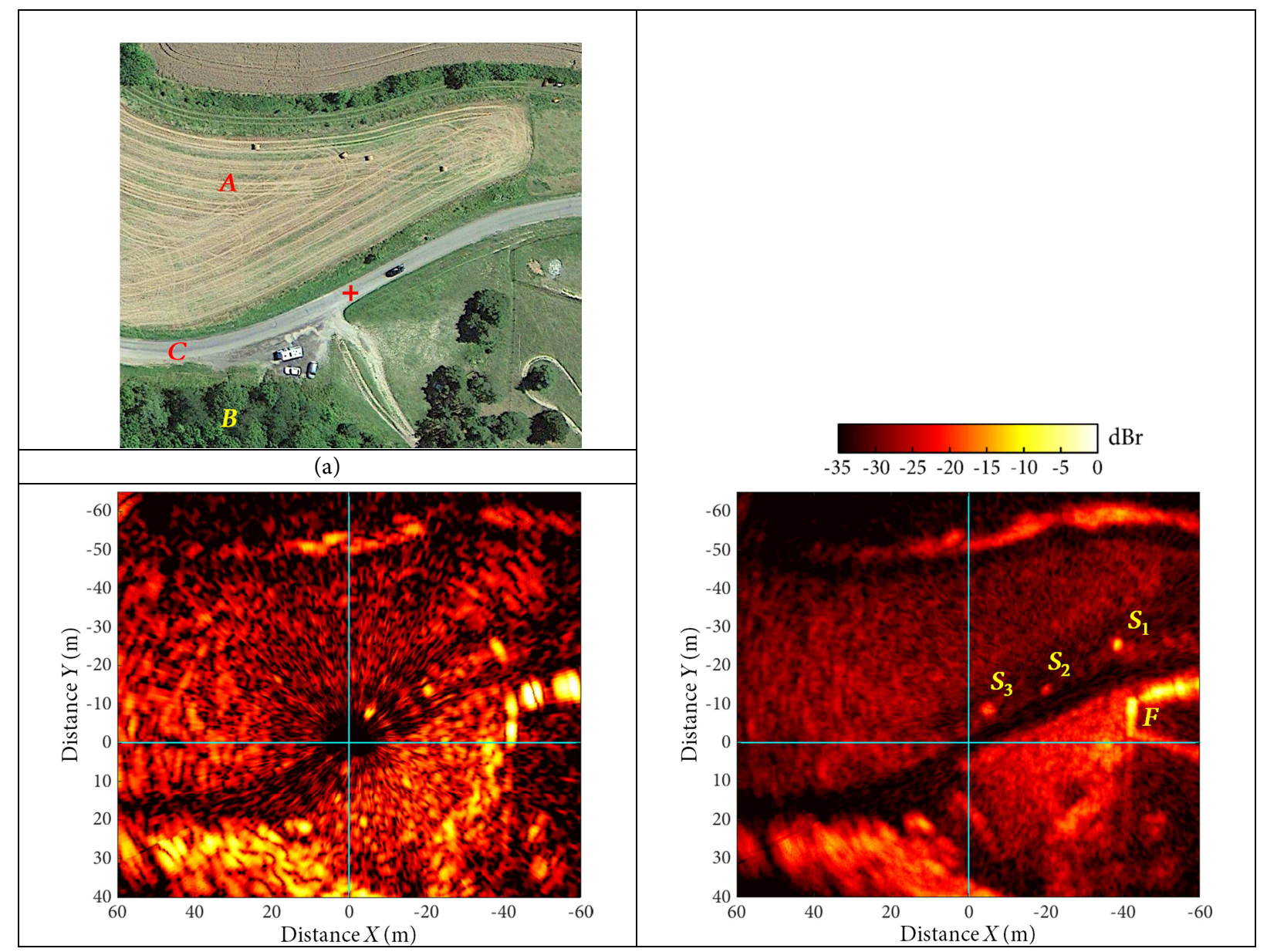

(b)

(c)

Figure 23: Illustration of Speckle effect and of Speckle filtering. (a) Aerial image of the test zone. Marks $A, B$ and $C$ indicate grassy area, vegetation area (trees) and paved road respectively. (b) Raw radar image in Cartesian coordinates. (c) Result of Speckle filtering. Marks $S_{1}, S_{2}$ and $S_{3}$ indicate a road sign and two shrubs respectively. Mark $F$. echo from a metallic fence.

One can observe that the Speckle noise is reduced. The grassy and vegetation areas appear more homogeneous. The paved road is darker because the backscattered signal is weaker due to a smaller surface roughness. Mark $S_{1}$ indicates the position of a road sign, $S_{2}$ and $S_{3}$ the presence of two shrubs. The strong echo localized with mark $F$ indicates the presence of a metallic fence.

\subsection{Results}

Radar data presented in Section 6.2.2, 6.2.3 and 6.2.4 are expressed in relative decibel (dBr). The value $\mathrm{O} \mathrm{dBr}$ is related to a maximum and arbitrary value of the backscattered radar signal. This value is common to all presented experimentations in order to allow comparison between the results obtained in the different environments.

\subsubsection{Multiplatform Implementation}

The size and weight of PELICAN radar facilitate its implementation on various kinds of vehicles. The main limitation is related to the position of radar on the vehicle: to be able to build panoramic representation of the surrounding environment, PELICAN radar must be positioned on the vehicle in such a way as to have an unobstructed view. Some examples of radar implementation are presented in Figure 24: robot, experimental vehicle (all-terrain vehicle) and boat. 


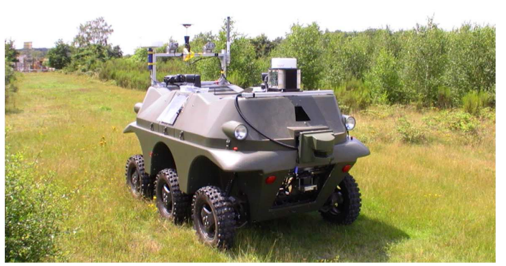

(a)

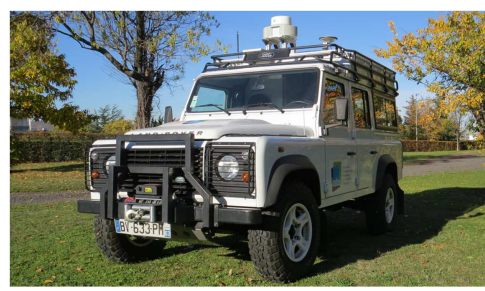

(b)

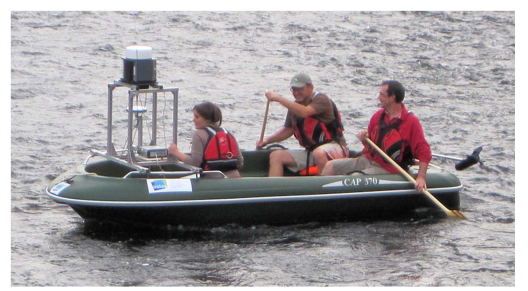

(c)

Figure 24: Examples of PELICAN radar positioning. (a) R-Trooper robot from Thales Company. (b) Irstea Institute experimental vehicle (all-terrain vehicle). (c) Boat of Irstea Institute.

PELICAN radar can be driven through the use of several interfaces (see Figure 25): laptop computer, portable touchscreen or smartphone application. The interfaces allow generating the commands to configure the radar, to control its functioning (start, stop, shut down, etc.), and to control the visualization or the storage of radar data.

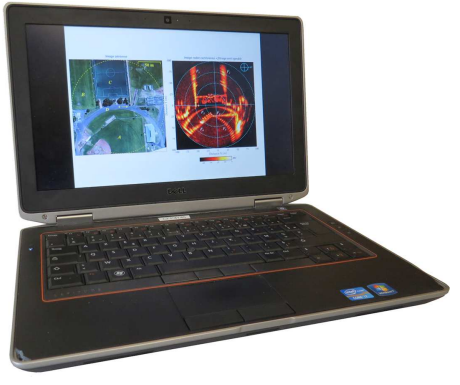

(a)

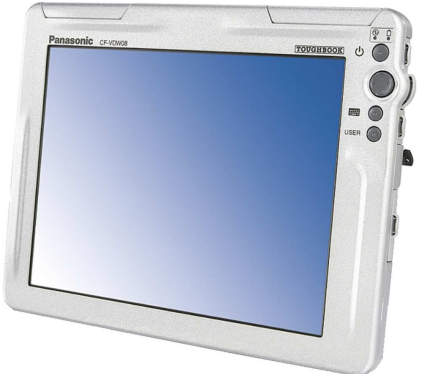

(b)

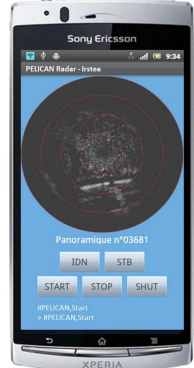

(c)

Figure 25: Control interfaces of PELICAN radar. (a) Laptop computer. (b) Portable touchscreen. (c) Smartphone application.

\subsubsection{Result in Urban Area}

The test area is a zone of economic activities located near Clermont-Ferrand, France. Figure 26(a) is an

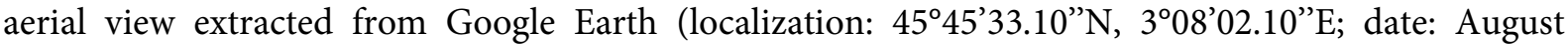
2013). Radar measurements were done in June 2016. The radar is positioned on the experimental vehicle from Irstea Institute presented in Figure 24(b), $3.3 \mathrm{~m}$ above ground. The vehicle mean velocity is $V=3.1 \mathrm{~m} / \mathrm{s}$. The vehicle is moving on a paved road, and it is positioned near an intersection. The cross shows its current position, and the arrow indicates the running direction. The dashed circle represents the maximum range of the radar. The main elements of the environment are buildings (concrete, metallic structures), light poles, street signs, cars and trees. The ground is composed of asphalt surfaces (roads, vehicle parking areas) and grassy areas.

Figure 26(b) is the raw image provided by PELICAN radar in polar coordinates, before Doppler shift and vehicle motion corrections, and before Speckle filtering. Radar spectra are computed with a 1024-point FFT and a Hann window function.

The radar image in Cartesian coordinates is presented in Figure 26(c) without Speckle filtering and with Speckle filtering in Figure 26(d). Both images include Doppler shift and vehicle motion corrections. It can be seen that metallic elements such as light poles $\left(P_{1}-P_{4}\right.$ marks), road signs $\left(S_{1}\right.$ and $S_{2}$ marks), or the walls of buildings ( $W_{1}$ and $W_{2}$ marks) are very efficient reflectors and they backscatter a high level of energy. In Figure 26(c), fading effects are illustrated with the echoes from the grassy areas or from the walls of buildings. The Speckle filtering in Figure 26(d) uses 10 successive panoramic images, and it allows reducing the salt-and-pepper pattern: for example, grassy areas appear more homogeneous and the walls are more continuous.

The 2D Richardson-Lucy deconvolution in Cartesian coordinates is presented in Figure 26(e) without Speckle filtering, with Speckle filtering in Figure 26(f). The number of iterations is 20. Both 
images include Doppler shift and vehicle motion corrections. One effect of the deconvolution process is to highlight strong echoes (metallic poles, walls, etc.) while reducing the amplitude of the ground clutter.

Specific radar distortions can be observed in the figures. For example, the echoes labelled $W_{1 \mathrm{~m}}$ and $W_{2 \mathrm{~m}}$ are ghost targets introduced by multipath effects: $W_{1 \mathrm{~m}}$ (respectively $W_{2 \mathrm{~m}}$ ) is the reflection of the wall labelled $W_{1}$ (respectively $W_{2}$ ), considering that the wall labelled $W_{2}$ (respectively $W_{1}$ ) is a mirror for the radar signals. Echoes labelled $M$ are a second illustration of multipath effects. Due to the specific geometry of the building, multibounce mechanisms occur: the echoes from the walls are delayed in range and several ghost targets are detected at increasing distances. The same phenomena can be observed in the lower left part of the images, where is located a building with a similar geometrical configuration.

\subsubsection{Result in Sport Field Area}

In this example, the radar is moving on a sports field. Figure $27(\mathrm{a})$ is an aerial view extracted from Google Earth (localization: $45^{\circ} 45^{\prime} 29.80^{\prime \prime} \mathrm{N}, 3^{\circ} 06^{\prime} 31.80^{\prime \prime} \mathrm{E}$; date: June 2012). Radar measurements were done in May 2016. PELICAN radar is positioned on the experimental vehicle from Irstea Institute presented in Figure 24(b), $3.3 \mathrm{~m}$ above ground. The vehicle mean velocity is $v=2.9 \mathrm{~m} / \mathrm{s}$. The vehicle is close to a baseball field (mark $A$ ) and close to football fields (marks $B$ and $C$ ). The vehicle is travelling on a paved road (mark $D$ ). The cross indicates the position of the vehicle, and the arrow the running direction. The dashed circle represents the maximum range of the radar. The sports field is an open environment, composed with large grassy areas, few trees, and metallic fences.

Figure 27(b) shows the raw image provided by PELICAN radar in polar coordinates, before Doppler shift and vehicle motion corrections, and before Speckle filtering. Radar spectra are computed with a 1024-point FFT and a Hann window function.

The radar image in Cartesian coordinates is presented in Figure 27(c) without Speckle filtering and with Speckle filtering in Figure 27(d). Both images include Doppler shift and vehicle motion corrections. It can be seen that the metallic elements, such as fences indicated with marks $F_{1}$ to $F_{6}$ in Figure $27(\mathrm{~d})$, backscatter a high level of energy. Fading effects are clearly visible in the grassy areas of Figure 27(c). The Speckle filtering in Figure 27(d) uses 10 successive panoramic images, and it allows reducing the salt-and-pepper pattern: for example, grassy areas appear more homogeneous.

The 2D Richardson-Lucy deconvolution in Cartesian coordinates is presented in Figure 27(e) without Speckle filtering, with Speckle filtering in Figure 27(f). The number of iterations is 20. Both images include Doppler shift and vehicle motion corrections. One effect of the deconvolution process is to highlight strong echoes (metallic fences and poles, trees, etc.) while reducing the amplitude of the ground clutter.

\subsubsection{Result in Natural Area}

In this example, the radar is moving in a natural area. Figure 28(a) is an aerial view extracted from Google Earth (localization: 4542'18.40"N, 300'43.70"E; date: August 2013). Radar measurements were done in June 2016. PELICAN radar is positioned on the experimental vehicle from Irstea Institute presented in Figure 24(b), $3.3 \mathrm{~m}$ above ground. The vehicle mean velocity is $v=3.1 \mathrm{~m} / \mathrm{s}$. The vehicle is travelling on a paved road, among several agricultural fields. The cross indicates the current position of the vehicle, and the arrow the running direction. The dashed circle represents the maximum range of the radar. The agricultural fields represent an open and poorly structured environment, with few obstacles: the major target is a vegetation hedge in the upper left part of the image (mark $H$ ). $A, B$ and $C$ marks represent the general configuration of the agricultural fields observed during the experimentation: $A$ is a natural grass field (height of grass: $0.5 \mathrm{~m}$ ); $B$ is a wheat 
field (height of wheat: $0.3 \mathrm{~m}$ ); and $C$ is a set of experimental plots of wheat (height of wheat: $0.5 \mathrm{~m}$ ) presenting a grid arrangement. It must be noticed that the aerial image has been registered in August 2013, also the configuration of the agricultural fields that can be seen in the image differs from that observed during the experimentation (in particular for the lower part of the image).

Figure 28(b) is the raw image provided by PELICAN radar in polar coordinates, before Doppler shift and vehicle motion corrections, and before Speckle filtering. Radar spectra are computed with a 1024-point FFT and a Hann window function.

The radar image in Cartesian coordinates is presented in Figure 28(c) without Speckle filtering and with Speckle filtering in Figure 28(d). Both images include Doppler shift and vehicle motion corrections. There are no strong reflectors such as metallic poles or buildings, and the average power backscattered by the environment is lower than that observed in Figure 26 and Figure 27. The radar image in Figure 28(c) is mainly characterized by a vegetation clutter and a strong Speckle effect. These random amplitude modulations are reduced by filtering in Figure 28(d). The Speckle filtering uses 10 successive panoramic images.

The 2D Richardson-Lucy deconvolution in Cartesian coordinates is presented in Figure 28(e) without Speckle filtering, with Speckle filtering in Figure 28(f). The number of iterations is 20. Both images include Doppler shift and vehicle motion corrections. The grid pattern introduced by the experimental plots can be observed in the lower left part of Figure 28(f). 
Author-produced version of the article published in Journal of Field Robotics, 2017, 1-27.

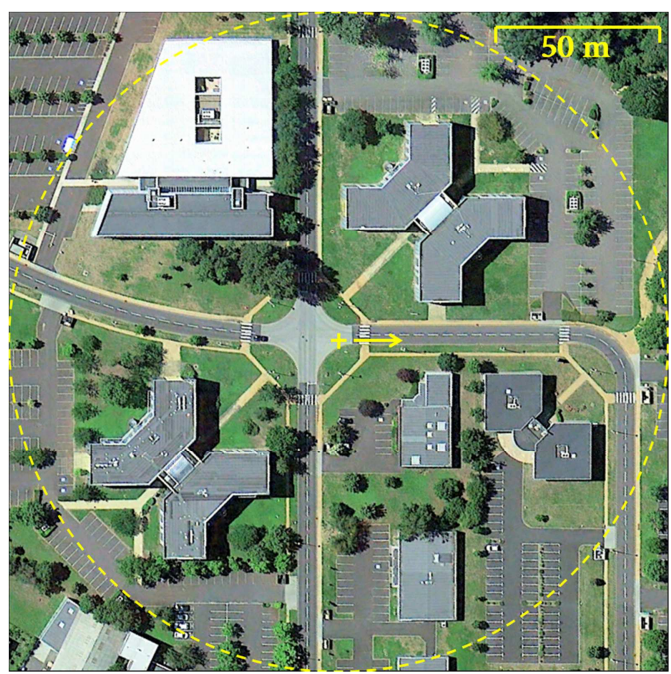

(a)

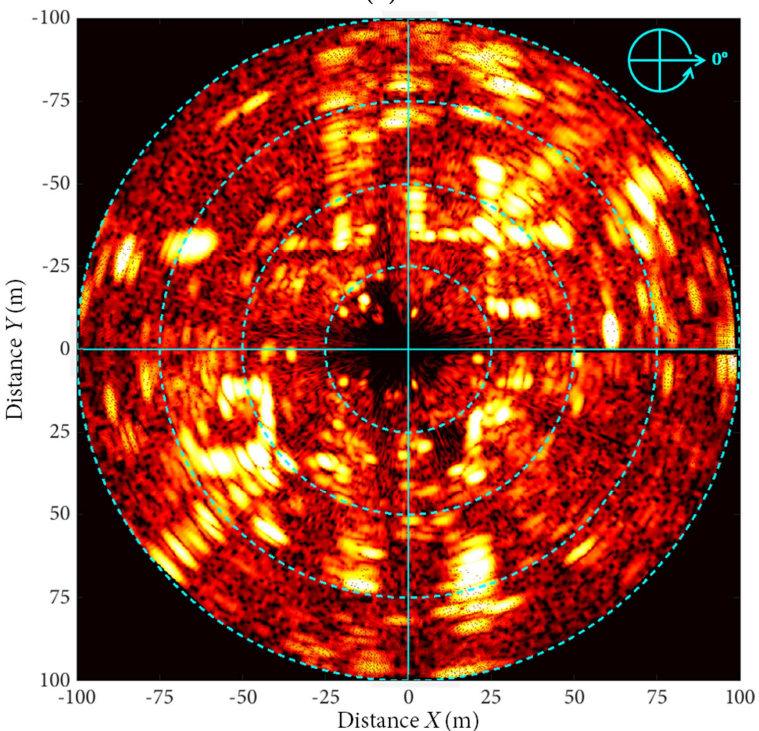

(c)

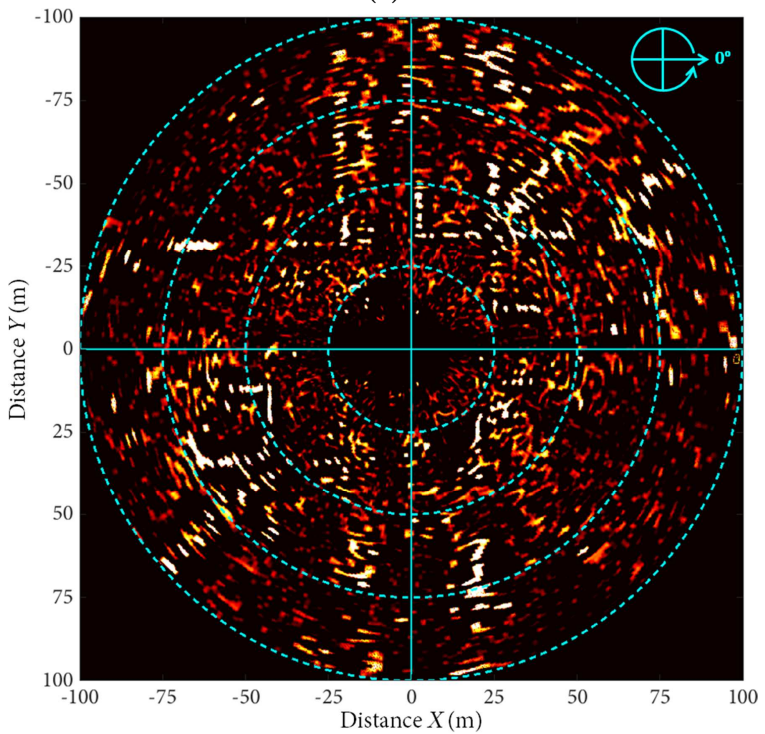

(e)
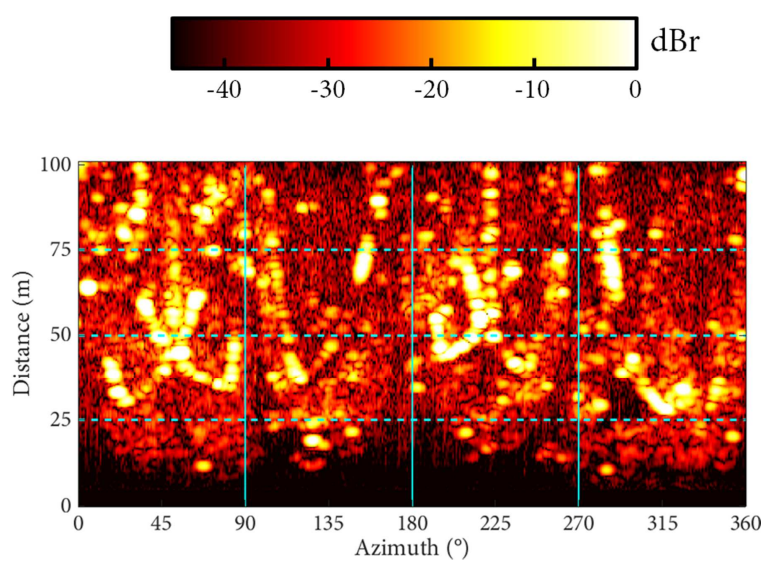

(b)

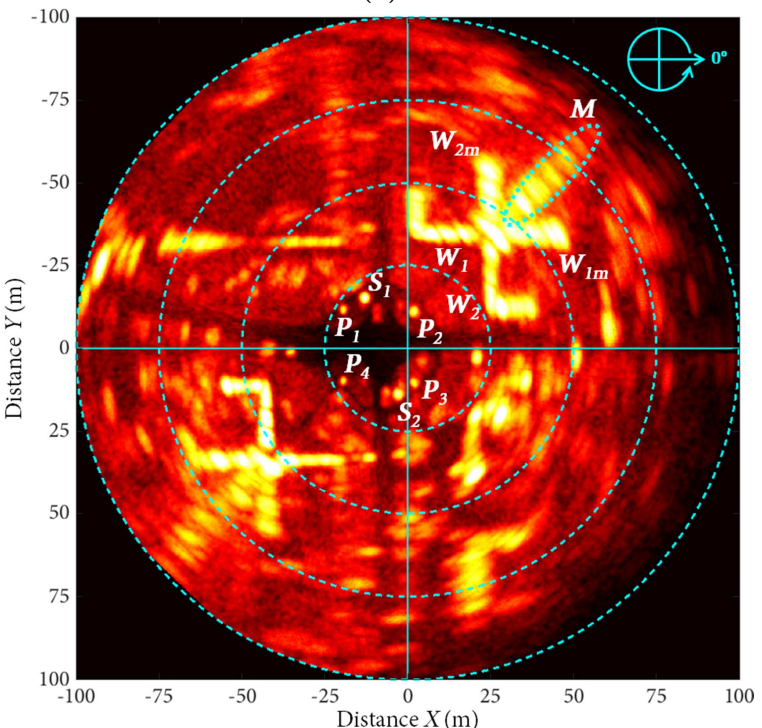

(d)

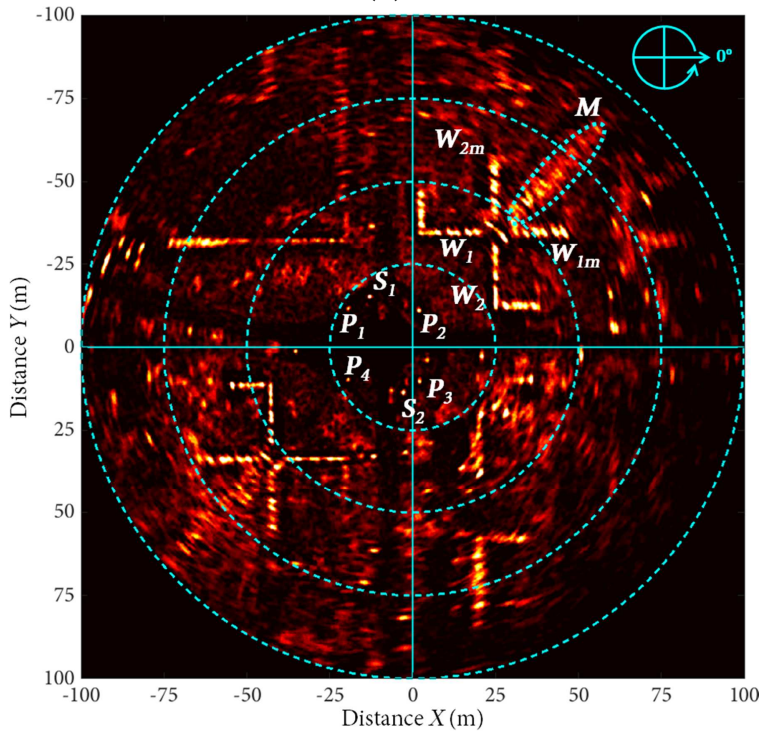

(f)

Figure 26: Experimental result in urban area. (a) Aerial image of the test zone. (b) Raw radar data in polar coordinates, computed with a 1024-point FFT. (c) and (d): radar images in Cartesian coordinates, without and with anti-speckle filter respectively. Result of the Richardson-Lucy deconvolution (e), with anti-speckle filter (f). 
Author-produced version of the article published in Journal of Field Robotics, 2017, 1-27.

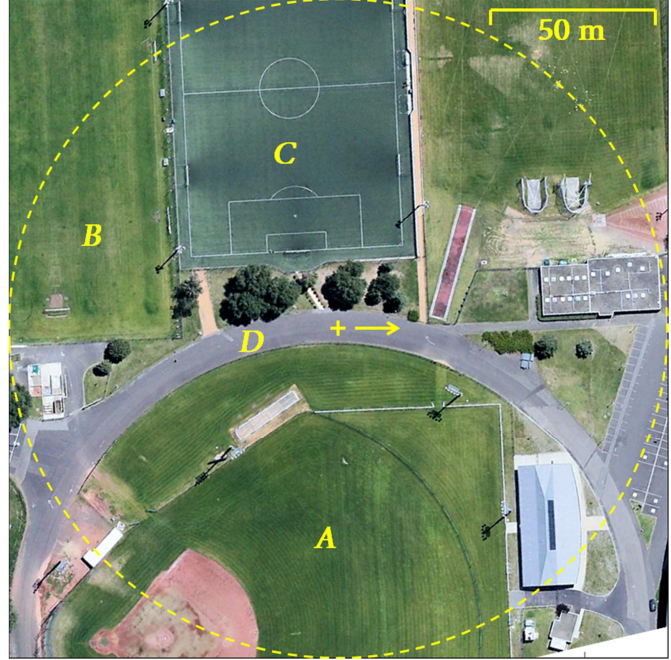

(a)

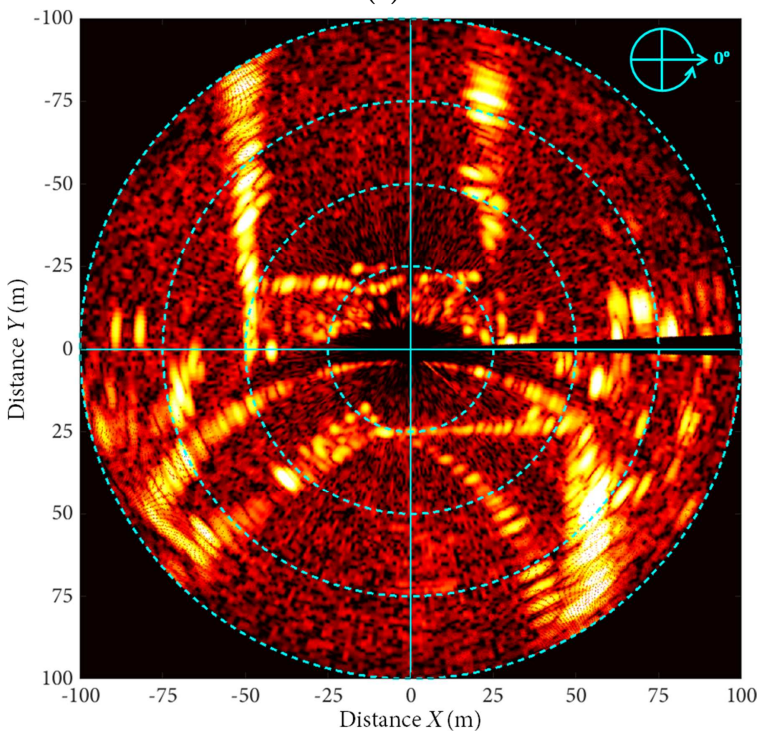

(c)

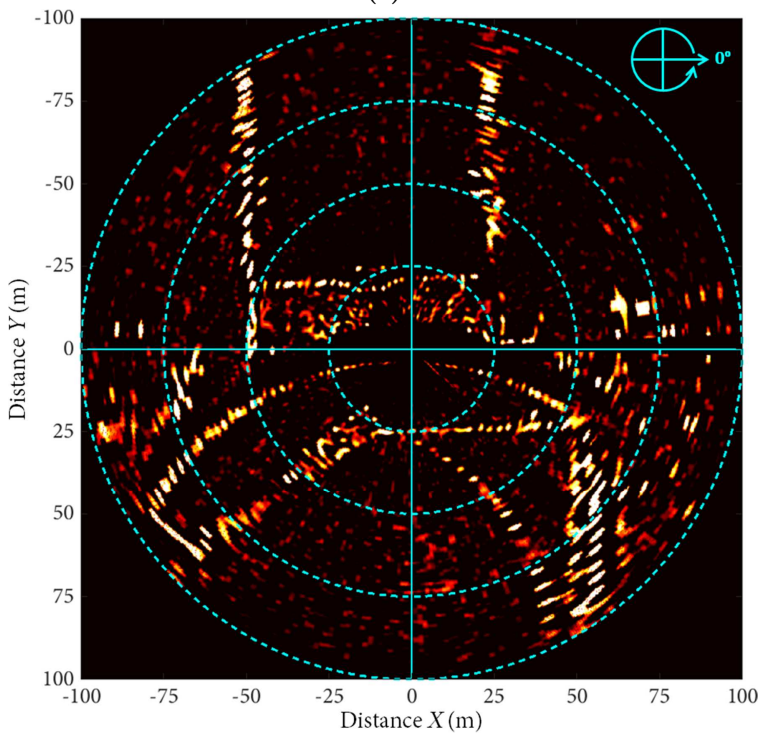

(e)
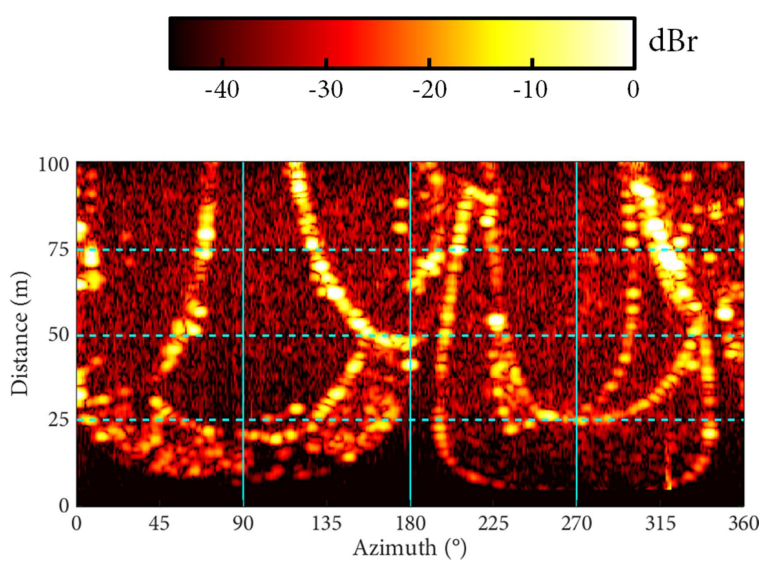

(b)

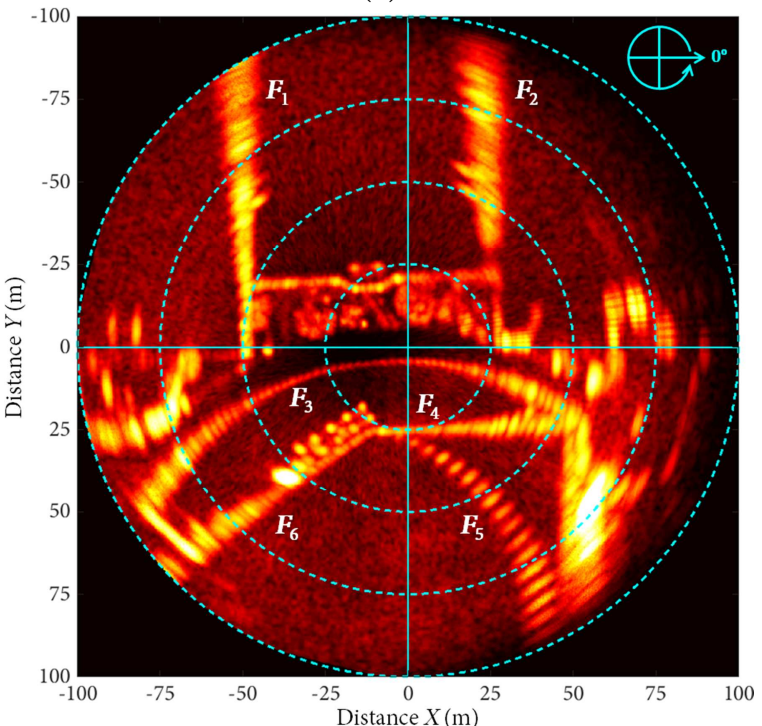

(d)

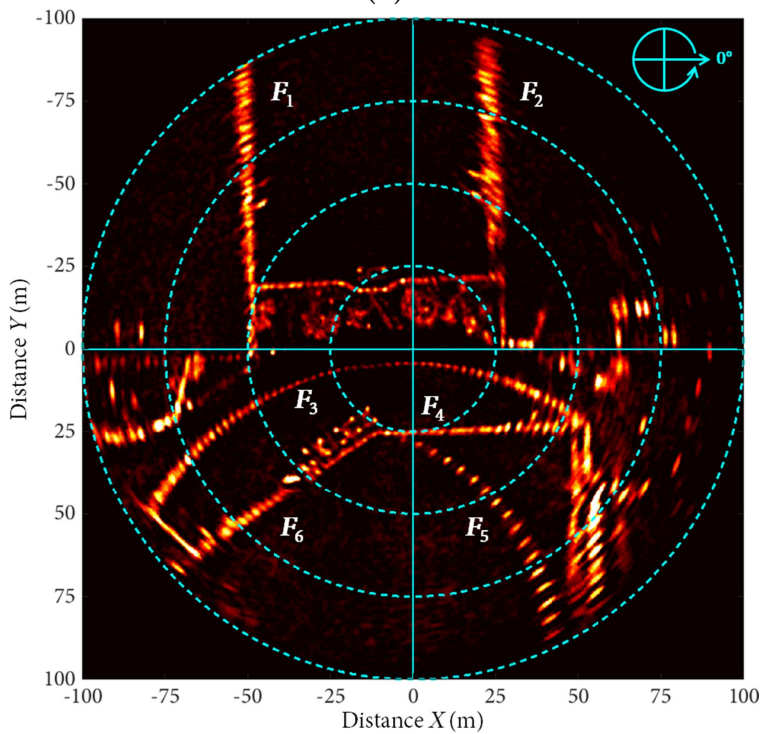

(f)

Figure 27: Experimental result in a sport filed area. (a) Aerial image of the test zone. Mark $A$ indicates a baseball field, $B$ and $C$ football fields, and $D$ a pavement area. (b) Raw radar data in polar coordinates, computed with a 1024-point FFT. (c) and (d): radar images in Cartesian coordinates, without and with anti-speckle filter respectively. Result of the Richardson-Lucy deconvolution (e), with anti-speckle filter (f). 
Author-produced version of the article published in Journal of Field Robotics, 2017, 1-27.

The original publication is available at http://onlinelibrary.wiley.com doi : 10.1002/rob.21770

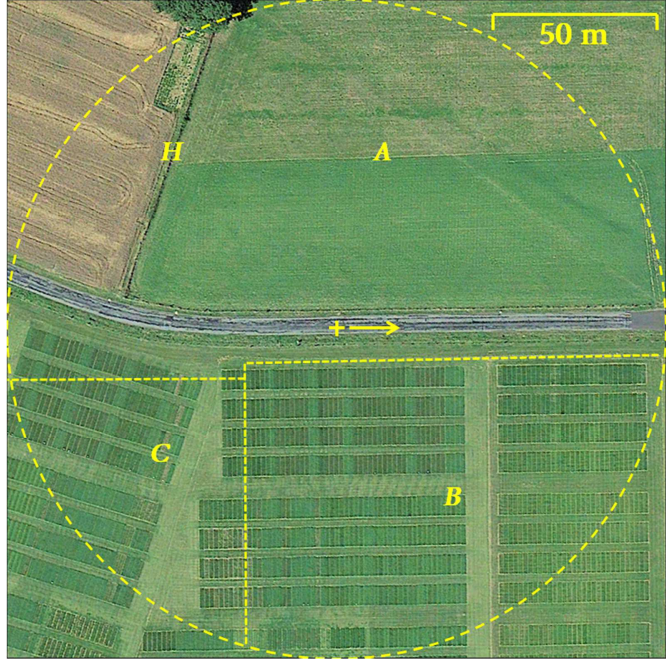

(a)

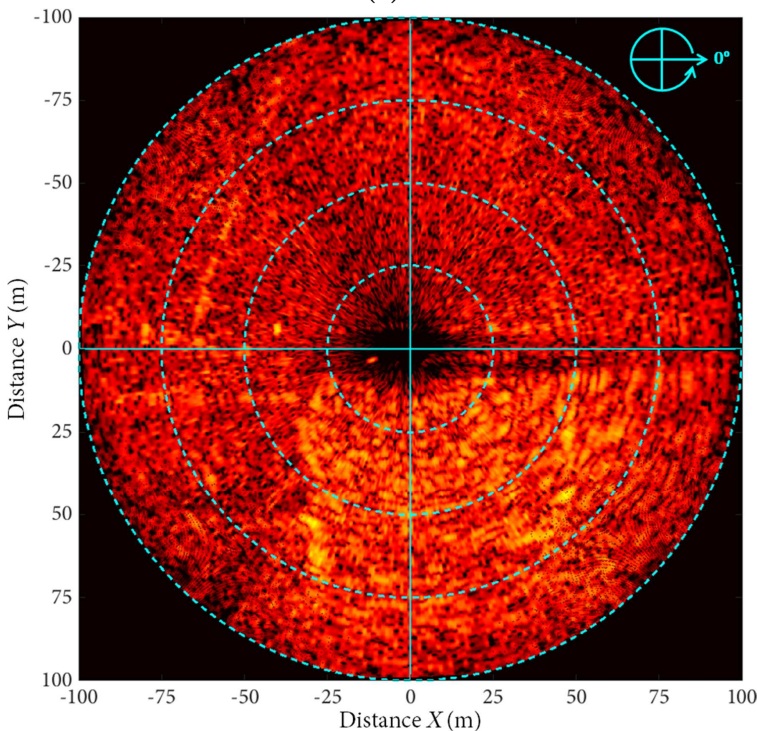

(c)

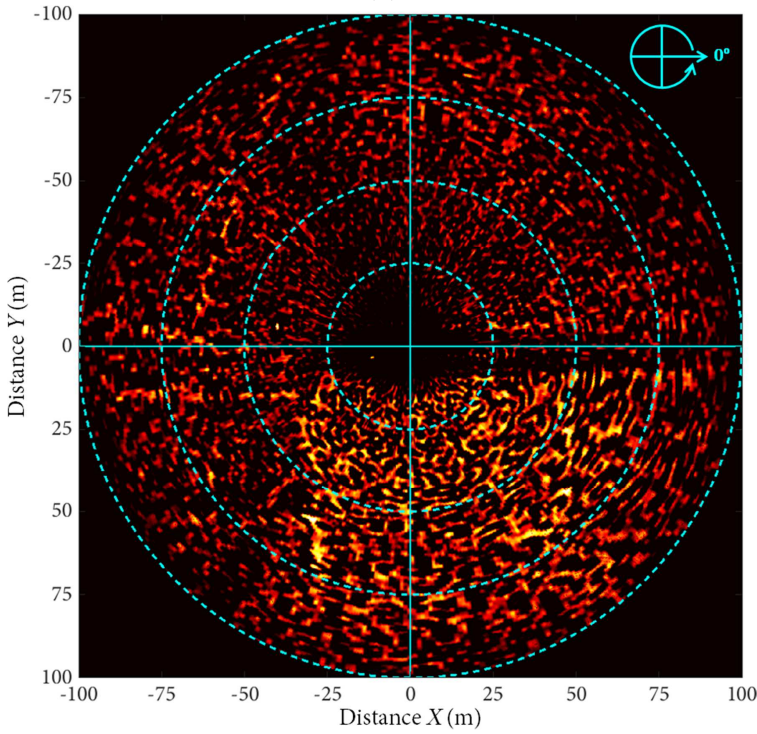

(e)
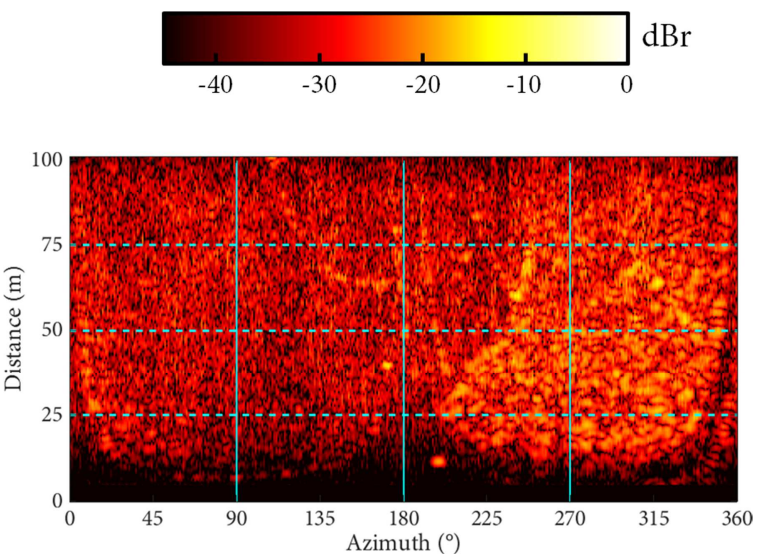

(b)

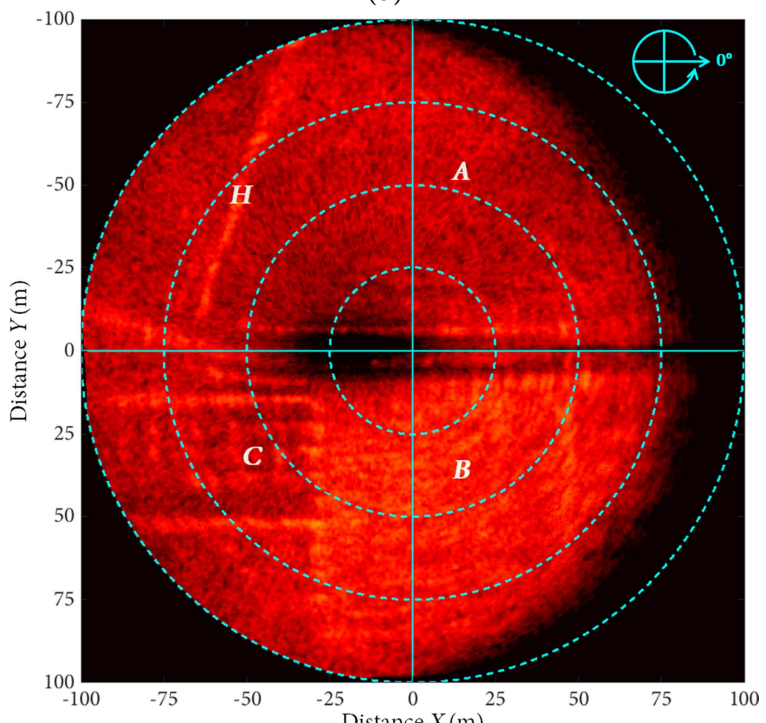

(d)

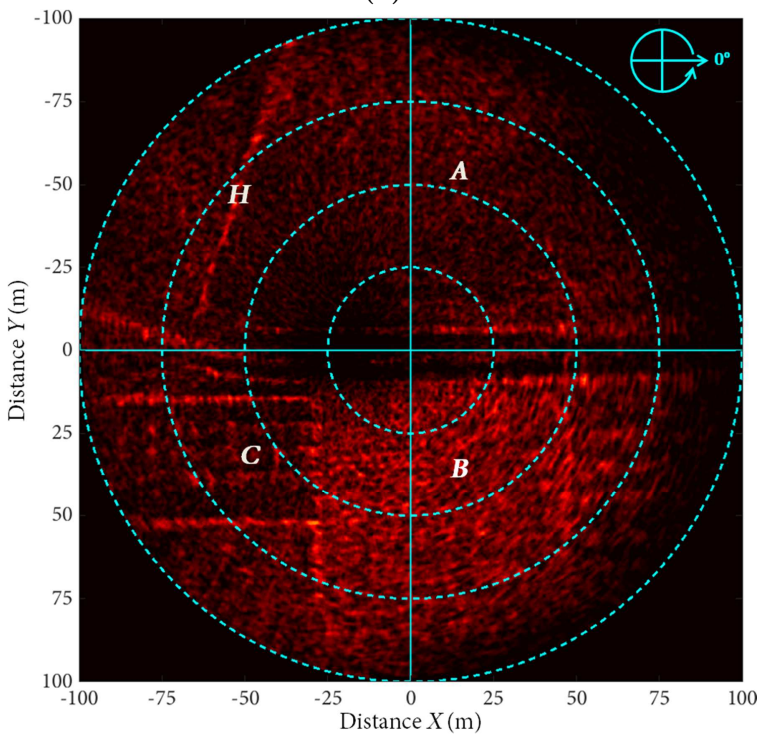

(f)

Figure 28: Experimental result in natural area. (a) Aerial image of the test zone. Mark $A$ indicates a grass field, $B$ a wheat field and $C$ a set of experimental plots of wheat. Mark $H$ indicates a vegetation hedge. (b) Raw radar data in polar coordinates, computed with a 1024-point FFT. (c) and (d): radar images in Cartesian coordinates, without and with anti-speckle filter respectively. Result of the Richardson-Lucy deconvolution (e), with anti-speckle filter (f). 


\section{Conclusion}

The objective of PELICAN radar is to provide a solution for robust perception within the framework of mobile robotics applications in outdoor and open environments. PELICAN radar is based on microwave radar technology, which highlights a low sensitivity to environment conditions such as weather events or dust. PELICAN is based on FMCW principles, and it is using a rotating antenna in order to build radar images of the surrounding environment over $360^{\circ}$. The solutions adopted for the hardware and software developments of PELICAN radar are presented in the paper: choice of a rotating fan-beam antenna; transmission of a microwave signal linearly modulated in frequency; filtering and amplification of the received signals; radar image construction; radar signal processing for angular and distance resolutions improvement based on 2D Richardson-Lucy deconvolution. The construction of radar images is illustrated with results obtained in several environments: urban, semiurban (sports field) and natural areas. Lightweight and small sized (6 kg, diameter $40 \mathrm{~cm}$ and height 24 $\mathrm{cm}$ for the 3D printer version), PELICAN radar can be easily positioned on various mobile terrestrial robots for obstacle detection and navigation applications. The radar is using a sawtooth modulation which offers a relative simplicity of hardware implementation, but which leads to a range-velocity ambiguity. To avoid the static environment assumption and to formally take into account mobile targets, new modulation laws are under development. In parallel, specific Simultaneous Localization And Mapping (SLAM) algorithms are developed in order to a build global map of the environment in which operates the robot.

\section{Access to PELICAN Radar Databases}

One of our goals in developing PELICAN radar has been to promote the use of microwave radars for perception in the "mobile robotics" community. In that sense, sets of PELICAN radar data are made available to researchers.

Each set includes all raw data acquired and stored during a PELICAN radar experiment: analog radar signal, antenna encoder, odometer, gyrometer and GPS.

Processed data are also provided: radar image in Cartesian coordinates, with and without 2D Richardson-Lucy deconvolution, with and without Speckle filtering.

Examples of Matlab ${ }^{\oplus}$ signal processing algorithms show the first steps to read, display and process radar data.

In order to access to PELICAN radar databases, please send an email request at pelican.radar@irstea.fr, indicating your name, title, email address, name of Institution, and a brief description of your interest in PELICAN radar data. You will receive in return an email with a download link. If you are interested in a scientific collaboration, to develop this technology or new applications in robotics domain, you can contact the corresponding author of the paper (raphael.rouveure@irstea.fr).

\section{Acknowledgments}

This study was supported by the Agence Nationale de la Recherche (ANR - the French national research agency) through the PELICAN research project (ANR-2010-EMMA-033). The PELICAN project was labeled by ViaMeca French pole of competitiveness. Researches were also supported by the French government research program "Investissements d'avenir" through the IMobS 3 Laboratory of Excellence (ANR-10-LABX-16-01). 


\section{Conflict of Interest}

The authors declare that there is no conflict of interest regarding the publication of this paper.

\section{References}

Abou-Khousa, M.A., Simms, D.L, Kharkovsky, S., \& Zoughi, R. (2009, May). High-resolution short-range wideband FMCW radar measurements based on MUSIC algorithm. In International Instrumentation and Measurement Technology Conference (I2MTC), Singapore.

Adams, M., \& Jose, E. (2006). Millimeter wave radar power-range spectra interpretation for multiple feature detection. In Autonomous mobile robot: sensing, control, decision making and applications (pp. 41-98). Boca Raton, FL: CRC Press.

Adams, M., Mullane, J., Jose, E., \& Vo, B.N. (2012). Robotic navigation and mapping with radar. Boston: Artech House.

Agence Nationale des Fréquences (2013). Tableau national de répartitions des bandes de fréquence. Edition de 2013.

Alvestegui, D.G.G. (2011). A linearization method for a UWB VCO-based chirp generator using dual compensation. Master Thesis, University of Kansas, KS.

Amazone (n.d.). BoniRob: Multipurpose field robot platform for agricultural applications. http://info.amazone.de/DisplayInfo.aspx?id=29417. Accessed November, 2017.

Argenti, F., Lapini, A., Bianchi, T., \& Alparone, L. (2013). A tutorial on speckle reduction in synthetic aperture radar images. IEEE Geoscience and Remote Sensing Magazine, 1(3), 6-35.

Bency, A., Lal, M.J., \& Abraham, T. (2013). High resolution spectral analysis useful for the development of radar altimeter. International Journal of Innovative Research in Electrical, Electronics and Control Engineering, $1(9), 407-412$.

Brooker, G., Hennessey, R., Bishop, M., Lobsey, C., Durrant-Whyte, H., \& Birch, D. (2006). High-resolution millimeter-wave radar systems for visualization of unstructured outdoor environments. Journal of Field Robotics, 23(10), 891-912.

Brooker, G., \& Jonhson, D. (2015). Low-cost millimeter wave imaging using a commercial plasma display. IEEE Sensors Journal, 15(6), 3557-3564.

Brooker, G., Jonhson, D., Underwood, J., Martinez, J., \& Xuan, L. (2015). Using the polarization of millimeterwave radar as a navigation aid. Journal of Field Robotics, 32(1), 3-19.

CARRE. (n.d.). ANATIS: Weeding Robot. http://www.carre.fr/en/crop-maintenance/weeding-robot/61anatis.html. Accessed November, 2017.

Carrington, W., Lynch, R., Moore, E., Isenberg, G., Fogarty, K., \& Fay, F. (1995). Superresolution threedimensional images of fluorescence in cells with minimal light exposure. Science, 268, 1483-1487.

Diewald, F., Klappstein, J., Dickmann, J., \& Dietmayer, K. (2011, June). An Adaption of the Lucy-Richardson Deconvolution Algorithm to Noncentral Chi-Square Distributed Data. In $12^{\text {th }}$ IAPR Conference on Machine Vision Applications, Nara, Japan.

El Natour, G., Ait-Aider, O., Rouveure, R., Berry, F., \& Faure, P. (2015). Toward 3D reconstruction of outdoor scenes using an MMW radar and a monocular vision sensor. Sensors, 15(10), 25937-25967.

Emmi, L., Gonzalez-de-Soto, M., Pajares, G., \& Gonzalez-de-Santos, P. (2014). New trends in robotics for agriculture: integration and assessment of a real fleet of robots. The Scientific World Journal, 2014, 1-21.

Ferretti, A., Monti-Guarnieri, A., Prati, C., Rocca, F., \& Massonnet, D. (2007). InSAR principles: guidelines for SAR interferometry processing and interpretation. Noordwijk, the Netherlands: ESA Publications Division, ESTEC.

Foessel, A., Bares, J., \& Whittaker, W. L. (2001, June). Three-dimensional map building with MMW radar. In International Conference on Field and Service Robotics, Helsinki, Finland.

Ford, J.P., Plaut, J.J., Weitz, C.M., Farr, T.G., Senske, D.A., Stofan, E.R., Michaels, G., \& Parker, T.J. (1993). Guide to Magellan Image Interpretation. Pasadena, CA: Jet Propulsion Laboratory Publication. 
Hasch, J., Topak, E., Schnabel, R., Zwick, T., Weigel, R., \& Waldschmidt, C. (2012). Millimeter-wave technology for automotive radar sensors in the $77 \mathrm{GHz}$ frequency band. IEEE Transactions on Microwave Theory and Techniques, 60(3), 845-860.

Hernandez-Gutierrez, A., Nieto, J.I., Bailey, T., \& Nebot, E.M. (2011, September). Probabilistic road geometry estimation using a millimeter-wave radar. In IEEE International Conference on Intelligent Robots and Systems, San Francisco, CA.

Heuel, S., \& Rohling, H. (2012, May). Pedestrian classification in automotive radar systems. In $19^{\text {th }}$ International Radar Symposium, Warsaw, Poland.

Heuer, M., Al-Hamadi, A., Rain, A., \& Meinecke, M.M. (2014, June). Detection and tracking approach using an automotive radar to increase active pedestrian safety. In IEEE Intelligent Vehicles Symposium, Dearborn, MI.

International Telecommunication Union (ITU) (2012). Radio Regulations - Articles. Edition of 2012. Geneva, Switzerland.

Jaud, M., Rouveure, R., Faure, P., Monod, M.O., \& Moiroux-Arvis, L. (2014, October). Correcting topography effects on terrestrial radar maps. In IEEE International Radar Conference (RADAR), Lille, France.

Jaud, M., Rouveure, R., Moiroux-Arvis, L., Faure, P., \& Monod, M.O. (2014). Boat borne radar mapping versus aerial photogrammetry and mobile laser scanning applied to river gorge monitoring. Open Journal of Remote Sensing and Positioning, 1, 48-63.

Johnson, D.G., \& Brooker, G. (2008). Wide band linearization of a millimetre-wave, linear frequency modulated radar employing a surface acoustic wave, delay line discriminator," In Smart Sensors and Sensing Technology. Berlin: Springer.

Lucy, L.B. (1974). An iterative technique for the rectification of observed distributions. The Astronomical Journal, 79(6), 745-754.

Milella, A., Reina, G., \& Underwood, J. (2015). A self-learning framework for statistical ground classification using radar and monocular vision. Journal of Field Robotics, 32, 20-41.

Milella, A., Reina, G., Underwood, J., \& Douillard, B. (2014). Visual ground segmentation by radar supervision. Robotics and Autonomous Systems, 62, 696-706.

Monod, M.O. (1995). Frequency modulated radar: a new sensor for natural environment and mobile robotics. Ph.D. Thesis, Paris VI University, France.

Mott, H. (2007). Remote Sensing with Polarimetric Radar - Appendix A: Fading and Speckle. Hoboken, NJ: John Wiley \& Sons, Inc.

Nathanson, F.E. (1999). Radar design principles: signal processing and the environment. New-York: McGrawHill International Editions.

Navtech. (2014). Navtech Radar W800-V Autonomous Vehicle Navigation and Obstacle Detection Sensor. http://cajunbot.com/wiki/images/6/64/Autonomous_vehicle_navigation.pdf. Accessed November, 2017.

Preza, C., Miller, M., Thomas, I., \& McNally, J. (1992). Regularized linear method for reconstruction of threedimensional microscopic objects from optical sections. Journal of the Optical Society of America A., 9, 219-228.

Reina, G., Jonhson, D., \& Underwood, J. (2015). Radar sensing for intelligent vehicles in urban environments. Sensors, 15(6), 14661-14978.

Reina, G., Milella, A., \& Rouveure, R. (2015, March). Traversability analysis for off-road vehicles using stereo and radar data. In IEEE International Conference on Industrial Technology, Seville, Spain.

Reina, G., Milella, A., \& Underwood, J. (2012). Self-learning classification of radar features for scene understanding. Robotics and Autonomous Systems, 60, 1377-1388.

Reina, G., Underwood, J., Brooker, G., \& Durrant-Whyte, H. (2011). Radar-based perception for autonomous outdoor vehicles. Journal of Field Robotics, 28(6), 894-913.

Richardson, W.H. (1972). Bayesian-based iterative method of image restoration. Journal of the Optical Society of America, 62(1), 55-59).

Rouveure, R. (2001). Short range soil characterization using microwaves techniques. Application to soil roughness measurement. Ph.D. Thesis, Clermont II University, France. 
Rouveure, R., Faure, P., Jaud, M., Monod, M.O., and Moiroux-Arvis, L. (2014, October). Distance and angular resolutions improvement for a ground-based radar imager. In IEEE International Radar Conference (RADAR), Lille, France.

Rouveure, R., Faure, P., \& Monod, M.O. (2016). PELICAN: panoramic millimeter-wave radar for perception in mobile robotics applications, Part 1: principles of FMCW radar and of 2D image construction. Robotics and Autonomous Systems, 81, 1-16.

Schmidt, R.O. (1986). Multiple emitter location and signal parameter estimation. IEEE Transactions on Antennas and Propagations, 34(3), 276-280.

Shahbazpanahi, S., Valaee, S., \& Bastani, M.H. (2001). Distributed source localization using ESPRIT algorithm. IEEE Transactions on Microwave Theory and Techniques, 49(10), 2169-2178.

Shanker, S.M., \& Arai, I. (2003). Signal Processing of ground penetrating radar using spectral estimation techniques to estimate the position of buried targets. EURASIP Journal on Advances in Signal Processing, $12,1198-1209$.

Shaw, P.J., \& Rawlins, D.J. (1991). The point-spread function of a confocal microscope: its measurement and use in deconvolution of 3-D data. Journal of Microscopy, 163(2), 151-165.

Skolnik, M.I. (1980). Introduction to radar systems. New-York: McGraw-Hill International Editions,

Slaughter, D.C., Giles, D.K., \& Downey, D. (2008). Autonomous robotic weed control systems: a review. Computers and Electronics in Agriculture, 61(1), 63-78.

Tokoro, S., Kuroda, K., Kawakubo, A., Fujita, K., \& Fujinami, H. (2003, June). Electronically scanned millimeterwave radar for pre-crash safety and adaptive cruise control system. In Intelligent Vehicles Symposium, Columbus, $\mathrm{OH}$.

Wang, T., Zheng, N., Xin, J., \& Ma, Z. (2011). Integrating millimeter wave radar with a monocular vision sensor for on-road obstacle detection applications. Sensors, 11(9), 8992-9008.

Widzyk-Capehart, E., Brooker, G., Scheding, S., Hennessy, R., Maclean, A., \& Lobsey, C. (2006, December). Application of millimetre wave radar sensor to environment mapping in surface mining. In International Conference on Control, Automation, Robotics and Vision (ICARCV), Singapore.

Zeng, S., \& Deng, W. (2015). Physics-based modelling method for automotive radar with frequency shift keying and linear frequency modulation. International Journal of Vehicle Design, 67(3), 237-258.

Zha, Y., Zhang, Y., Huang, Y. \& Yang, J. (2015). Bayesian angular superresolution algorithm for real-aperture imaging in forward-looking radar. Information, 6(4), 650-668. 